ARTICLE

\title{
Structural basis for microtubule recognition by the human kinetochore Ska complex
}

\author{
Maria Alba Abad ${ }^{1, \star}$, Bethan Medina ${ }^{1, \star}$, Anna Santamaria ${ }^{2, \star}$, Juan Zou1 ${ }^{1}$ Carla Plasberg-Hill, \\ Arumugam Madhumalar ${ }^{3}$, Uma Jayachandran, Patrick Marc Redli ${ }^{2}$, Juri Rappsilber ${ }^{1,4}$, \\ Erich A. Nigg ${ }^{2} \&$ A. Arockia Jeyaprakash
}

The ability of kinetochores (KTs) to maintain stable attachments to dynamic microtubule structures ('straight' during microtubule polymerization and 'curved' during microtubule depolymerization) is an essential requirement for accurate chromosome segregation. Here we show that the kinetochore-associated Ska complex interacts with tubulin monomers via the carboxy-terminal winged-helix domain of Ska1, providing the structural basis for the ability to bind both straight and curved microtubule structures. This contrasts with the Ndc80 complex, which binds straight microtubules by recognizing the dimeric interface of tubulin. The Ska1 microtubule-binding domain interacts with tubulins using multiple contact sites that allow the Ska complex to bind microtubules in multiple modes. Disrupting either the flexibility or the tubulin contact sites of the Ska1 microtubule-binding domain perturbs normal mitotic progression, explaining the critical role of the Ska complex in maintaining a firm grip on dynamic microtubules.

\footnotetext{
${ }^{1}$ Wellcome Trust Centre for Cell Biology, Institute of Cell Biology, University of Edinburgh, Michael Swann Building, Kings Buildings, Mayfield Road, EH9 3JR Edinburgh, UK. ${ }^{2}$ Biozentrum, University of Basel, Klingelbergstrasse 50/70, CH-4056 Basel, Switzerland. ${ }^{3}$ National Institute of Immunology, Aruna Asaf Ali Marg, New Delhi 110067, India. ${ }^{4}$ Department of Biotechnology, Technische Universität Berlin, 13353 Berlin, Germany. ${ }^{\star}$ These authors contributed equally to this work. Correspondence and requests for materials should be addressed to A.A.J. (email: jeyaprakash.arulanandam@ed.ac.uk).
} 
E stablishment of physical connections between the chromosomes and the spindle microtubules (MTs) via the kinetochore $(\mathrm{KT})$ is essential for faithfully segregating the duplicated chromosomes to daughter cells ${ }^{1,2}$. A key property of the functional KT is its ability to maintain attachments to the plus end of MTs, as they undergo cycles of polymerization and depolymerization commonly known as dynamic instability ${ }^{3-6}$. As MT depolymerization contributes to the force required for driving chromosome segregation ${ }^{7-10}$, MT-binding factors that can stay attached to and/or track depolymerizing MTs are essential. At the outer $\mathrm{KT}$, a protein interaction network called the KMN network (consisting of the protein KNL1 and the protein complexes Mis12 and $\mathrm{Ndc} 80$ ) provides the direct binding site for MTs ${ }^{5,11}$. Among these components, the $\mathrm{Ndc} 80$ complex is the major MT-binding factor, whereas KNL1 also possesses MT-binding ability ${ }^{11,12}$.

The Ndc80 complex is a heterotetramer composed of Ndc80, Nuf2, Spc24 and Spc25. The globular domains of Ndc80 and Nuf2 are connected to the globular domains of Spc24 and Spc25 via a long coiled-coil structure, resulting in an $\sim 60$-nm dumbbell-shaped architecture ${ }^{5,13,14}$. Although the globular heads of Spc24 and Spc25 mediate the KT association, those of Ndc80 and Nuf2 directly interact with MTs $5,13,15,16$. The Ndc80 complex can track depolymerizing MTs when attached to microspheres and it influences MT dynamics by stabilizing straight $\mathrm{MTs}^{17,18}$. In budding yeast, a ten-subunit protein complex called Dam1 can form a ring around MTs and cooperates with the Ndc80 complex in maintaining stable $\mathrm{KT}$ attachments to dynamic $\mathrm{MTs}^{19-22}$. However, no obvious structural Dam1 homologue has been identified in metazoans.

Originally discovered in a proteomics screen ${ }^{23}$, the Ska complex is now recognized as a key element required for maintaining stable KT-MT attachments ${ }^{24-29}$. The ternary Ska complex, composed of Ska1, Ska2 and Ska3, localizes to the outer $\mathrm{KT}$ in a KMN-dependent manner. There, it is regulated by the Aurora B kinase ${ }^{30}$, much like the Dam1 complex ${ }^{19,20}$. Cells depleted of the Ska complex fail to maintain stable KTMT attachments, resulting in chromosome congression failure followed by cell death ${ }^{25,26}$. The Ska complex can directly interact with MTs and track depolymerizing MTs in vitro ${ }^{26,31}$. Accordingly, it has been proposed that the Ska and Ndc80 complexes form an integrated MT-binding assembly ${ }^{31}$. The ability of the Ska complex to track depolymerizing MTs in vitro and its dependency on the KMN for its localization and function suggest that the Ska complex may be a functional equivalent of the Dam/DASH complex in metazoans ${ }^{25,26}$. Besides stabilizing KT-MT attachments, the Ska complex has also been implicated in silencing the spindle checkpoint ${ }^{24,27,32}$.

During MT growth and shrinkage, MTs undergo important conformational changes. Protofilaments adopt a curved conformation during MT depolymerization and a straight conformation during polymerization. Understanding the structural basis for how the $\mathrm{Ndc} 80$ and Ska complexes interact with dynamic MTs is indispensable for understanding the mechanistic aspects of KT-MT attachments. Structural characterizations of Ndc80-MT interactions have shown that the Ndc80 complex binds MTs by interacting at the dimeric interface of $\alpha$ - and $\beta$-tubulins. This mode of interaction is also thought to influence the plus-end dynamics of $\mathrm{MTs}^{33,34}$. Although no atomic structure of the Dam1 complex is available, electron microscopy studies have provided insight into how multimeric Dam1 complexes assemble into a ring-like structure encircling MTs ${ }^{19,35}$. Our previous work demonstrated that the Ska complex is a dimer of triple helical bundles formed by Ska1, Ska2 and Ska3, resulting in a $\mathrm{W}$-shaped structure with a maximum interatomic distance of $\sim 350 \AA$ (ref. 36). The MT-binding domains (MTBDs) of the Ska complex protrude at the ends of the W-shaped homodimer, suggesting a transversal mode of MT binding at the KT-MT interface ${ }^{36}$. A recently reported nuclear magnetic resonance (NMR) structure of the Caenorhabditis elegans Ska1MTBD revealed the involvement of a winged-helix domain in MT recognition ${ }^{31}$. At this point, information on how the Ska complex interacts with MTs is crucial to understand the role of the Ska complex in potentially coupling MT dynamics and chromosome segregation. By combining X-ray crystallography, crosslinking/ mass spectrometry (MS) and biochemistry, we have here characterized the MTBD of the human Ska complex and evaluated its interaction with MTs in vitro and in vivo. We show that the Ska complex, unlike the Ndc80 complex, can bind tubulin monomers in different orientations via its multiple MT contact sites, allowing it to recognize MTs in a conformation-independent manner. These results provide novel structural and functional insights into the role of the Ska complex in maintaining stable attachments to dynamic MTs.

\section{Results}

Ska1 $_{92-255}$ is essential for correct mitotic progression. We previously demonstrated that the C-terminal domains of Ska1 (Ska1 $\left.{ }_{92-255}\right)$ and Ska3 (Ska3 $\left.{ }_{102-412}\right)$ are essential for the function of the Ska complex ${ }^{36}$. Deletion of Ska1 ${ }_{92-255}$ completely abolished the MT-binding ability of the complex, supporting its role in recognizing $\mathrm{MTs}^{36}$. Secondary structure predictions suggested that Ska1 ${ }_{92-255}$ possesses a globular domain preceded by an unstructured region of 40 amino acids. Proteolysis experiments using trypsin and MS analysis identified a stable fragment of Ska1 encompassing residues 133-255 (Ska1 ${ }_{133-255}$; Fig. 1a). Consistent with our previous work, Ska1 $1_{133-255}$ showed weak MT binding on its own ${ }^{17}$ (Fig. 1b). As the full-length (FL) Ska complex is a dimer, we asked whether dimerization of $\mathrm{Ska1}_{133-255}$ would increase its affinity for MTs. Exploiting the propensity of glutathione $S$-transferase (GST) to dimerize, we tested the ability of GSTfused Ska1 $1_{133-255}$ to bind MTs. Although GST-Ska1 133-255 is not strictly equivalent to the FL Skal dimer in the native complex, and thus might bind MTs differently, it clearly interacted with MTs more efficiently than Ska1 $1_{133-255}$ (Fig. 1b). These observations suggest that $\mathrm{Skal}_{133-255}$ (from now on referred to as MTBD) is the major MT-binding element within the Ska complex, which, on dimerization, can bind MTs more efficiently.

We next evaluated the requirement of the Ska1-MTBD and the loop (Ska1 ${ }_{92-132}$, referred to as Skal loop) connecting the aminoterminal helical domain $\left(\mathrm{Ska1}_{1-91}\right)$ for correct mitotic progression. As reported earlier ${ }^{25,27,31}$, Skal depletion resulted in a significant increase in mitotic timing (Fig. 1c; see Supplementary Fig. S1a for depletion efficiency). A majority of cells showed metaphase-like appearance (Fig. 1d and Supplementary Movie 1), but roughly a third of the cells showed prolonged prometaphase with chromosome congression defects (Supplementary Movie 2), probably reflecting a more complete depletion of the Ska complex $^{25,30}$. FL mCherry-Skal efficiently rescued depletion of endogenous Ska1 (Supplementary Movie 3) and cells progressed through mitosis comparably with control (GL2-treated) cells (Fig. 1c). Replacement of endogenous Ska1 by Ska1 $1_{1-132}$ failed to rescue normal progression through mitosis (Fig. 1c): onset of anaphase was delayed and the frequency of apoptosis increased, but most of the cells showed proper chromosome alignment with timings comparable to cells rescued with Skal wild type (WT; Fig. 1d, Supplementary Table S1 and Supplementary Movie 4). Moreover, Ska1 $1_{1-132}$ localized to KTs but failed to decorate spindle MTs and showed no bundling activity when compared with Ska1 WT (Supplementary Fig. S1b,c). These observations are 
a

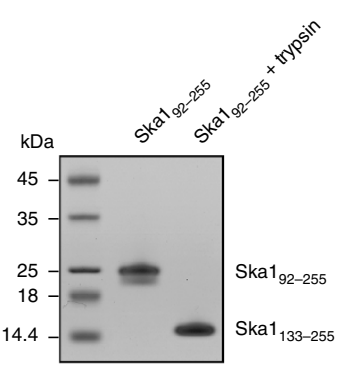

b
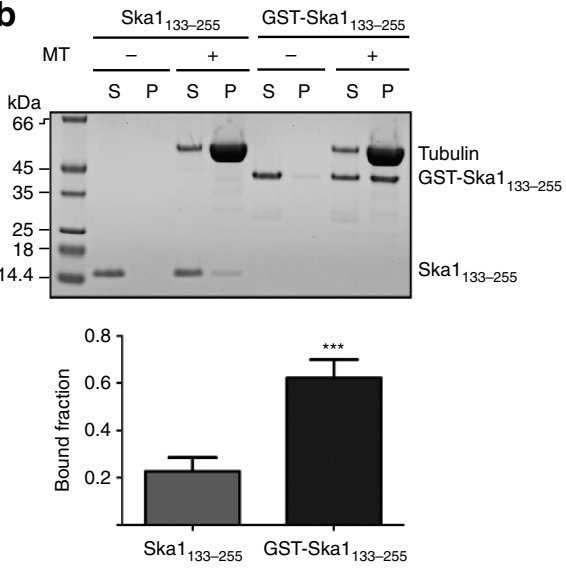

C

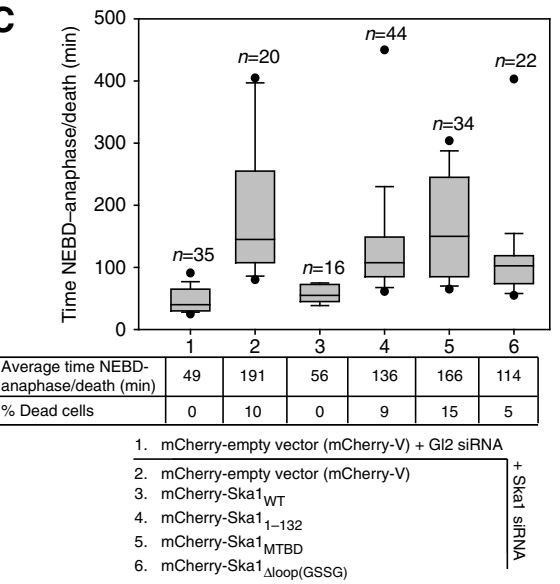

d

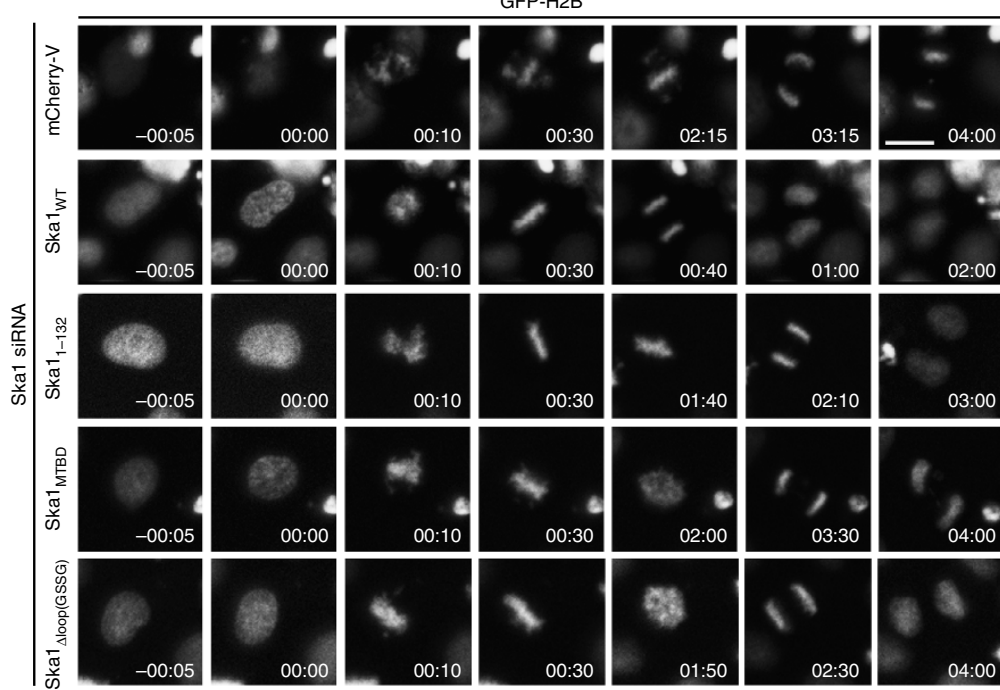

e

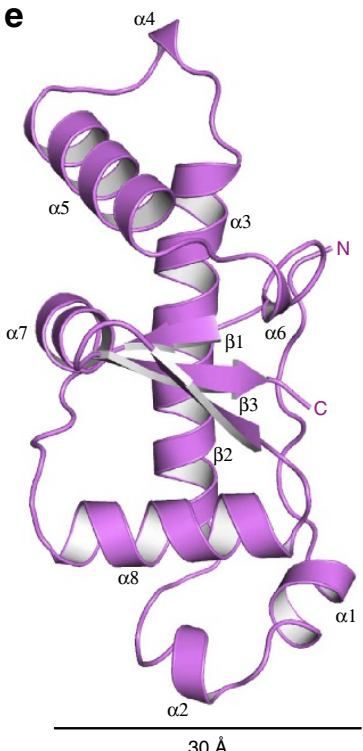

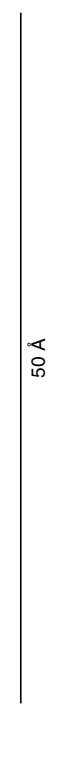

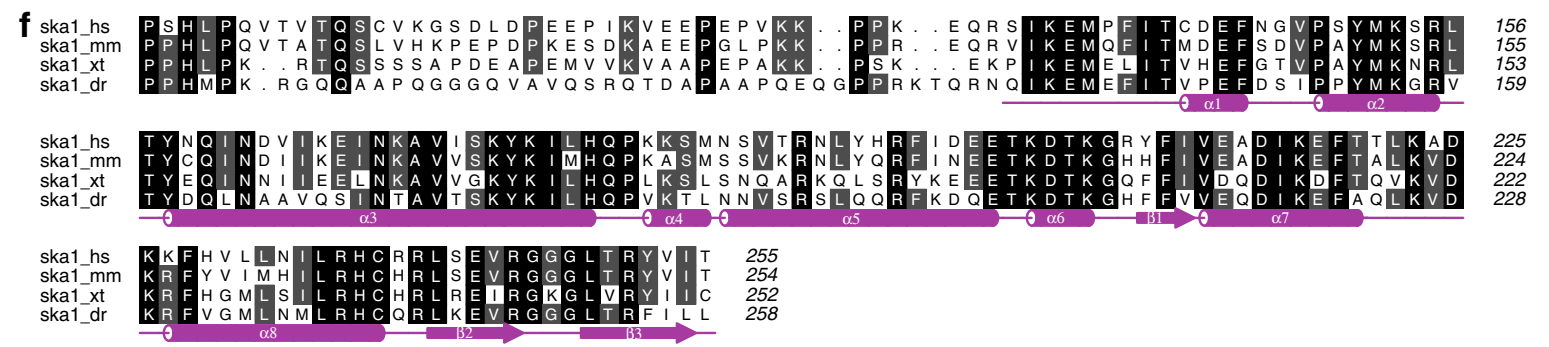

Figure 1 | Characterization of functional determinants of Ska1. (a) Limited proteolysis of the $S_{k a 1} 1_{92-255}$ with trypsin that led to the formation of a stable fragment identified by MS as Ska1 ${ }_{133-255}$. Uncropped scan of the gel is shown in Supplementary Fig. S8a. (b) Top, representative SDS-PAGE of cosedimentation assays comparing the MT-binding activity of $\mathrm{Ska1}_{133-255}$ and GST-Ska1 ${ }_{133-255}$. Bottom, quantification of the MT-binding assays in $\mathbf{b}$ (mean $\pm \mathrm{s} . \mathrm{d}$., $n=4$,

${ }^{\star \star \star} P \leq 0.001$, $t$-test). (c) Box-and-whisker plot showing the elapsed time ( $\mathrm{min}$ ) between nuclear envelope breakdown (NEBD) and anaphase onset/death for individual cells. The total number of cells ( $n$ ) from two or more independent experiments is given above each box. Lower and upper whiskers represent 10th and 90th percentiles, respectively. Table summarizing information from the live cell experiments shown below regarding the average time in mitosis (from NEBD until anaphase onset/cell death) and the percentage of cells dying in mitosis. (d) Representative stills from time-lapse video-microscopy experiments illustrating mitotic progression of HeLa S3 cells stably expressing histone H2B-GFP treated as in c. Time in h:min is indicated. $T=0$ was defined as the time point at which NEBD became evident. Scale bar, $10 \mu \mathrm{m}$. (e) Cartoon representation of the structure of human Ska1-MTBD, which possesses a modified winged-helix domain with an elongated shape. The length of the structure is $\sim 50 \AA$ whereas the width is $\sim 30 \AA$. Secondary structure elements are labelled. (f) Sequence alignment of human Ska1 11-255 showing amino acid conservation between H. sapiens (hs), Mus musculus (mm), Xenopus tropicalis (xt) and Danio rerio (dr). Secondary structure elements are shown below the aligned sequences. Amino acid conservation is highlighted in grey.

consistent with a previous report ${ }^{31}$ and show the requirement of the Ska1-MTBD for ensuring stable KT-MT interactions and timely mitotic progression, although this domain seems dispensable for initial chromosome alignment. In contrast, replacement of WT Skal by Ska1-MTBD resulted not only in delayed anaphase onset and problems in maintaining a tight metaphase plate (Fig. 1c and Supplementary Movie 5) but also increased the time for initial chromosome alignment 
(Supplementary Table S1). Considering that Ska1-MTBD lacks both the helical domain required for intermolecular interactions involving Ska2 and Ska3, and the loop that precedes it, these results highlight the importance of the N-terminal helical domain and the Skal loop for the complete functionality of the complex (Supplementary Fig. S1). Ska1-MTBD decorated the mitotic spindle but failed to localize to KTs (Supplementary Fig. S1b). The removal of amino acids 1-132 of Skal promoted the nuclear localization of the Ska1-MTBD, possibly through a nuclear export signal encompassing amino acids $52-58$ of Skal (ref. 37). This precluded the assessment of the MT-bundling ability of this construct in vivo (Supplementary Fig. S1c).

We next sought to specifically evaluate the functional requirement of the Skal loop. Transfection of Ska1 $\Delta$ loop(GSSG), where amino acids 92-132 were replaced by a short linker sequence (GSSG), delayed both chromosome alignment and anaphase onset by twofold (Fig. 1c, Supplementary Table S1 and Supplementary Movie 6). It is to be noted that the Ska1$\Delta$ loop(GSSG) can bind MTs with comparable efficiency to the WT Ska complex in in vitro MT-binding assays (Supplementary Fig. S1d). Interestingly, the bulk of the Ska1 $\Delta$ loop(GSSG) mutant showed no or weak KT localization, suggesting a potential role for the loop in mediating intermolecular interactions required for KT localization (Supplementary Fig. S1b). Together, these results suggest that the flexibility associated with the loop region (and/or intermolecular interactions mediated by it) is required for timely progression through mitosis.

Ska1-MTBD possesses a modified winged-helix motif. To understand the structural basis for the ability of human Ska complex to bind MTs, we obtained crystals of the Ska1-MTBD in two different crystal forms that diffracted X-rays to about $2 \AA$ (Table 1). The structure was determined by single anomalous dispersion (SAD) experiments using crystals obtained from selenomethionine-incorporated samples. Models from crystal form I (space group C222 1 ) and II (space group P $3_{2}$ ) were refined to 2.1 and $2.3 \AA$ with $R$ factors of 19.6 and 22.5 , and $R_{\text {free }}$ factors of 25.1 and 26.8, respectively (Table 1 ), and superpose well with an overall root mean square deviation of $0.6 \AA$. Residues $133-142$ are stabilized in an extended conformation, followed by eight $\alpha$ helical segments $(\alpha 1-\alpha 8)$ and a C-terminal $\beta$-hairpin (Fig. 1e,f). The structural analysis showed that the Ska1-MTBD is related to a winged-helix domain, a domain known for its ability to bind DNA and in mediating protein-protein interactions ${ }^{38-42}$. The Ska1-MTBD differs from the canonical winged-helix domain by the incorporation of two additional modules (Fig. 1e and Supplementary Fig. S2a).

Human and C. elegans Ska1-MTBDs show structural variations. During the course of this work, the NMR structure of the MTBD of the C. elegans Skal was reported ${ }^{31}$. The Ska1-MTBD of C. elegans shares $28 \%$ sequence identity and $46 \%$ sequence similarity with its human counterpart. Structural comparisons show that the overall topology of the human and C. elegans Ska1MTBDs is the same (structures superpose with an overall root mean square deviation of $3.0 \AA$ ). However, in the $C$. elegans structure, helices $\alpha 5$ and $\alpha 8$, and the $\beta$-strands $\beta 2$ and $\beta 3$ are in a different orientation relative to the rest of the structure, resulting in noticeable changes in the surface charge distribution of the MTBD (Supplementary Fig. S2b-d). Structure-based sequence alignment reveals that $C$. elegans amino acid Thr168 acts as a hinge residue between $\alpha 3$ and $\alpha 5$ (Supplementary Fig. S2c). In contrast, the corresponding amino acid in higher vertebrates is proline (182, human numbering), an amino acid that has limited backbone conformational flexibility (Supplementary Fig. S2c).

\begin{tabular}{|c|c|c|}
\hline & Crystal form 1 & Crystal form 2 \\
\hline \multicolumn{3}{|l|}{ Data collection } \\
\hline Space group & $\mathrm{C} 222_{1}$ & $\mathrm{P}_{2}$ \\
\hline \multicolumn{3}{|l|}{ Cell dimensions } \\
\hline$a, b, c(\AA)$ & $39.01,161.58,104.48$ & $47.18,47.18,116.50$ \\
\hline$\alpha, \beta, \gamma\left({ }^{\circ}\right)$ & $\begin{array}{c}\text { 90, } 90,90 \\
\text { Peak }\end{array}$ & $90,90,120$ \\
\hline Wavelength & 0.98 & 1.541 \\
\hline Resolution $(\AA)$ & $63.9-2.0(2.12-2.01)$ & $58.3-2.3(2.37-2.25)$ \\
\hline$R_{\text {merge }}$ & $7.8(45.5)$ & $4.8(30.8)$ \\
\hline$|/ \sigma|$ & $15.2(3.8)$ & $17.3(3.6)$ \\
\hline Completeness (\%) & $99.8(99.6)$ & $98.5(90.1)$ \\
\hline Redundancy & $7.5(7.5)$ & $4.5(4.0)$ \\
\hline \multicolumn{3}{|l|}{ Refinement } \\
\hline Resolution $(\AA)$ & $27.0-2.1$ & $58.3-2.3$ \\
\hline No. of reflections & 169,025 & 60,423 \\
\hline$R_{\text {work }} / R_{\text {free }}$ & $19.4 / 24.6$ & $22.5 / 26.8$ \\
\hline No. of atoms & 2,161 & \\
\hline Protein & 2,026 & 1,962 \\
\hline Water & 135 & 40 \\
\hline \multicolumn{3}{|l|}{$\beta$-Factors } \\
\hline Protein & 52.9 & 68.2 \\
\hline Water & 50.5 & 50.3 \\
\hline \multicolumn{3}{|c|}{ Root mean square deviation } \\
\hline Bond lengths $(\AA)$ & 0.008 & 0.008 \\
\hline Bond angles $\left(^{\circ}\right)$ & 1.09 & 1.12 \\
\hline
\end{tabular}

To uncover potential differences in the properties of human versus $C$. elegans Ska1-MTBDs, as manifested by sequence and structural variations, $50 \mathrm{~ns}$ molecular dynamic simulations (MDS) were carried out using the two structures. The analysis of the root mean squared fluctuations of the $\mathrm{C} \alpha$ atoms during MDS shows that the MTBD domain of $C$. elegans Skal has more intrinsic structural flexibility (particularly regions that show conformational variation) than its human counterpart (Supplementary Fig. S2e). Considering the modest sequence similarity between the human and C. elegans Ska1-MTBDs, the suggested conformational variability in the respective structures seems reasonable. However, it is to be noted that the structures we are comparing were obtained by crystallography and NMR, respectively; thus, definitive conclusions on the proposed structural variations require further validation.

Ska interacts with MTs using a multipartite mode of binding. The basic nature of the Ska1-MTBD (predicted $\mathrm{pI}=9.2$ ) led us to hypothesize that the Ska complex recognizes MTs through electrostatic interactions. Analysis of the electrostatic surface potential revealed the existence of contiguous positively charged patches all over the Ska1-MTBD surface (Fig. 2a), suggesting the potential involvement of multiple MT contact sites. Of the 23 Lys (K)/Arg (R) residues that are present in the Ska1-MTBD, 14 are clearly exposed to solvent (Fig. 2a). To identify the critical residues required for MT binding, K/R residues that cluster on the surface were mutated to Ala (A) in the context of the FL human Ska complex. Before subjecting the mutants to MT cosedimentation assays, we analysed their size-exclusion chromatographic profiles to rule out the influence of the mutations on the overall structure of the complex. All the mutants behaved identically to the WT Ska complex, suggesting that mutations do not affect the proper folding of the Ska complex (Supplementary Fig. S3c). Although mutations at K170/177 and K135/203/206 

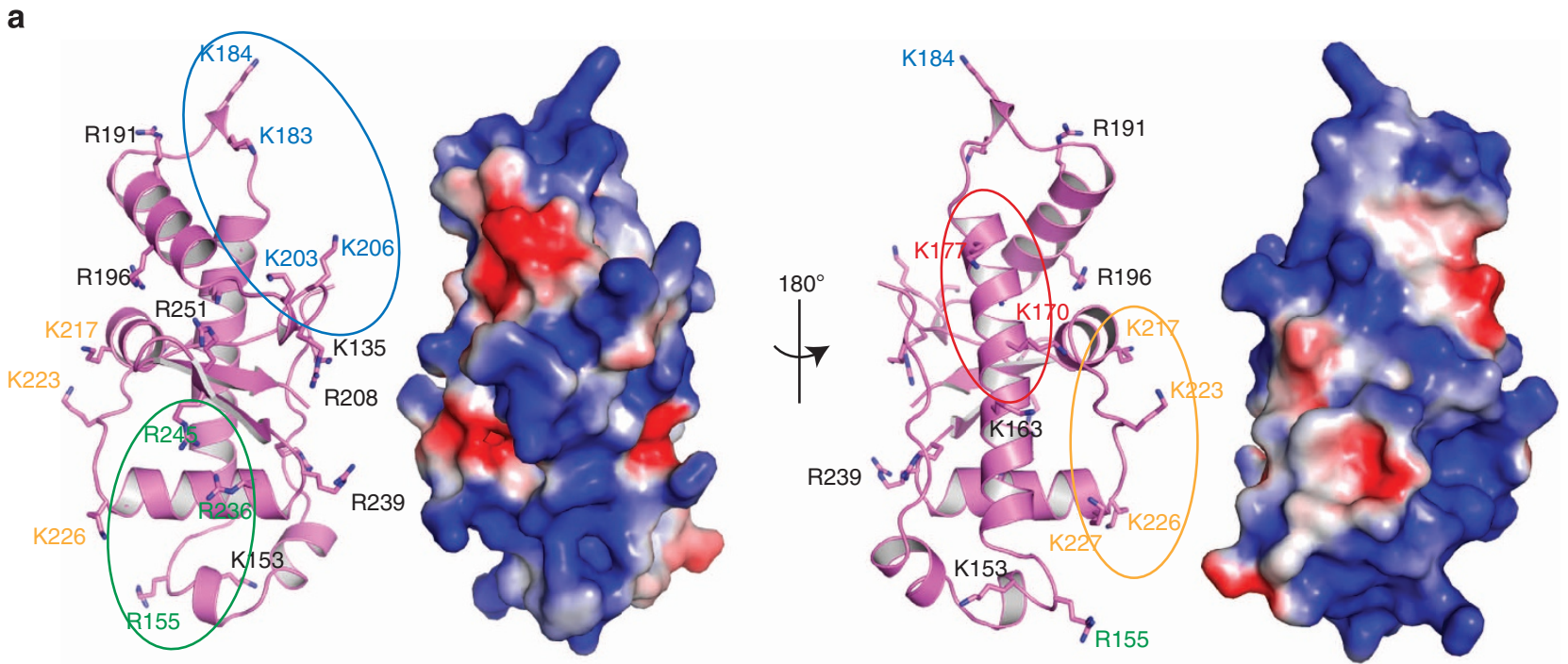

b

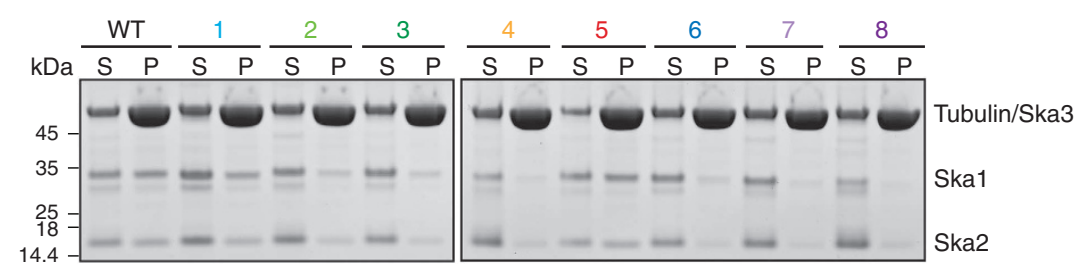

1 K135/203/206A; 2 R236/245A; 3 R155/236/245A; 4 K217/223/226/227A; 5 K170/177A; 6 K183/184/203/206A; 7 (6+4) K183/184/203/206/217/223/226/227A;

$8(7+2)$ K183/184/203/206/217/223/226/227/R236/245A
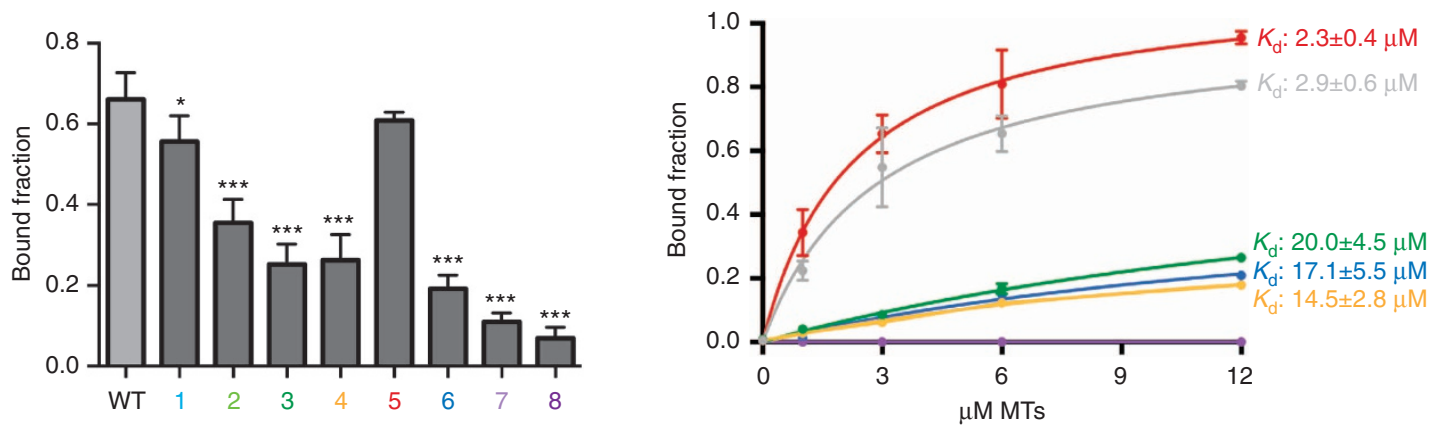

WT K170/177A R155/236/245A K217/223/226/227A K183/184/203/206A K183/184/203/206/217/223/226/227/R236/245A

Figure 2 | The Ska complex binds MTs through multiple positively charged clusters. (a) Cartoon representation of the Ska1-MTBD where surfaceexposed K/R residues are shown as sticks (left). Surface representation of the Ska1-MTBD in the same orientation with electrostatic surface potential revealing the presence of positively charged patches (right). Residues clustered based on their proximity and mutated to A to test their involvement in MT recognition are highlighted in different colours. (b) Cosedimentation assays of the different K/R- to A-untagged Ska mutants were performed. Representative gels (upper panel) and quantifications of MT cosedimentation assays (bottom panel). Concentration of Ska1 mutants and MTs used in the assays are $3 \mu \mathrm{M}$ and $6 \mu \mathrm{M}$, respectively (mean \pm s.d., $n \geq 3,{ }^{\star} P \leq 0.05,{ }^{\star \star \star} P \leq 0.001 ; t$-test; right bottom panel). $K_{d}$ values were calculated using $1 \mu \mathrm{M}$ Ska and 0-12 $\mu \mathrm{M}$ MTs (bottom left panel). Uncropped scans of the gels are shown in Supplementary Fig. S8b.

showed no major effects on MT binding, mutations at R155/236/ 245 , a region reported recently to be critical for MT binding ${ }^{31}$, together with two new regions identified in this study, K183/184/ 203/206 and K217/223/226/227, all showed significant reductions in MT binding $\left(K_{\mathrm{d}}=20 \pm 4.5,17.1 \pm 5.5\right.$ and $14.5 \pm 2.8 \mu \mathrm{M}$, respectively, versus $\mathrm{WT}=2.9 \pm 0.6 \mu \mathrm{M})$, pointing to the existence of multiple MT interaction sites (Fig. 2b and Supplementary Fig. S3a). Confirming this notion, a combination of R236/245A with R155A resulted in stronger reduction in MT binding than R236/245A alone (Fig. $2 \mathrm{~b}$ and Supplementary Fig. S3b), and the simultaneous mutation of multiple clusters (K183/184/203/206/217/223/226/227/R236/ 245A) almost completely abolished MT binding (Fig. 2b). These results provide clear evidence that the human Ska complex binds MTs through a multipartite binding mode of Ska1-MTBD.

Multipartite MT binding is required for Ska complex function. We next evaluated the functional significance of the positively charged clusters of the Ska1-MTBD using small interfering RNA (siRNA) rescue assays with the above $\mathrm{K}$ or $\mathrm{R}$ to $\mathrm{A}$ mutants. In line with in vitro results, cells transfected with K170/177A and K135/ 
203/206A mutants showed normal mitotic progression (Fig. 3). Similar to WT cells, cells transfected with K170/177A kept the ability to strongly bundle MTs (Supplementary Fig. S4a), highlighting the preserved MT-binding activity in this mutant. Transfection of the Ska1 K135/203/206A mutant resulted in fewer and weaker MT bundles in interphase cells (Supplementary Fig. S4a), most likely to be reflecting a role of K203/206 in MT binding (Fig. 2b); as shown below, this becomes apparent in combination with mutations at other residues (see below). In contrast, rescues by the Ska1 K183/184/203/206A, R155/236/ $245 \mathrm{~A}$ and K217/223/226/227A mutants resulted in perturbed mitotic progression, characterized by a prolonged delay in anaphase onset and an increase in the number of apoptotic cells, but no MT bundling in interphase cells (Fig. 3, Supplementary Fig. S4a and Supplementary Table S2). In agreement with the results of the in vitro MT-binding assay, the R236/245A mutant on its own showed a milder phenotype, but when combined with R155A it showed a much more pronounced phenotype (Fig. 3), suggesting cooperation between R236/245A and R155A.

All Ska1 mutants tested were able to form a complex with Ska2 and Ska3 (Fig. 2b), and they localized to KTs, indistinguishable from WT Ska1 (Supplementary Fig. S4b), indicating that the mitotic defects described above are due to interference with MT binding. Indeed, the majority of cells expressing R155/236/245A, K217/223/226/227A and K183/184/203/206A contained aligned chromosomes (Fig. 3b, Supplementary Table S2 and Supplementary Movies 7-9, respectively), reminiscent of Ska1-MTBD expression (see above) and indicative of the requirement of the MT-binding activity for robust KT-MT attachments, but not for the initial contact between KTs and MTs.

Ska1 interacts with tubulin monomers at multiple sites. Having established a multipartite mode of MT binding by the Ska complex, we next aimed at identifying the structural features of MTs that are recognized by the Ska complex. For this purpose, we crosslinked the Skal-MTBD/Ska complex with MTs, using 1-ethyl-3-[3-dimethylaminopropyl] carbodiimide hydrochloride (EDC). This reagent crosslinks $\mathrm{K}$ (and less favourably $\mathrm{S}, \mathrm{T}, \mathrm{Y}$ ) to $\mathrm{E}$ or D. Analysis of the crosslinked products in SDSpolyacrylamide gel electrophoresis (SDS-PAGE) showed a predominant band that migrated at the expected molecular weight for one Ska1-MTBD/Skal crosslinked to an $\alpha$-/ $\beta$-tubulin monomer (marked by asterisks in Supplementary Fig. S5a,b).

MS analysis of the crosslinked products allowed us to pinpoint the residues involved in intermolecular recognition between the Ska complex and tubulins (Fig. 4 and Supplementary Fig. S5e). The overall sequence coverage for Ska1/Ska1-MTBD and tubulin monomers was almost complete, except for the flexible C-terminal tails of tubulin (Supplementary Fig. S5f). Consistent with our biochemical and functional analyses, most of the crosslinks observed for the FL Ska complex bound to MTs involved the MTBD of Ska1. The Ska1-MTBD made almost identical crosslinks with MTs, regardless of whether it was analysed on its own or in the context of the Ska complex, highlighting the specificity of the interaction (Fig. 4a,b). Furthermore, Ska2, which has been shown not to have any MT-binding activity, did not produce any crosslinked peptides with tubulin, confirming the specificity of the crosslinking reaction. Among the three K/R clusters that we identified to be crucial for MT binding and function, two (K183/184/203/206 and K216/217/223/226) showed crosslinks with tubulin monomers. Mapping of the crosslinked residues on the three-dimensional structures of Ska1MTBD and MTs showed that these clusters contact globular/ folded regions of tubulin monomers (unlike most MT-binding proteins that interact with MTs by recognizing acidic tails of tubulins) mainly at two helices: $\mathrm{H} 3$ and $\mathrm{H} 4$ of $\beta$-tubulin and $\mathrm{H} 3$ and $\mathrm{H} 12$ of $\alpha$-tubulin (Fig. 4c). Interestingly, intermolecular contacts of Ska1 with $\mathrm{H} 4$ of $\beta$-tubulin and $\mathrm{H} 12$ of $\alpha$-tubulin seem to be sequence specific, as these tubulin residues are unique to $\alpha$ - and $\beta$-isoforms (results not shown).

To rule out the possibility that the crosslinking peptides observed are due to nonspecific interactions with free tubulin monomers, we pelleted MTs crosslinked to Ska1-MTBD before MS analysis and compared the results with those obtained from non-pelleted samples (Fig. 4). Analysis of both samples by SDS-PAGE showed identical crosslinked products (Supplementary Fig. S5g). Furthermore, the contact sites observed were almost identical in both pelleted and non-pelleted samples (Supplementary Fig. S5h), attesting to the specificity of the interactions.

In this analysis, we did not detect crosslinks involving the R155/236/245 cluster. This, as well as the fact that we did not detect acidic tails of tubulin monomers, may be due to the following technical reasons: first, EDC, the crosslinking reagent used in this study, does not crosslink arginines; second, peptides derived from the acidic tails of tubulins may have escaped detection by MS possibly because of the presence of posttranslational modifications (notably polyglutamylation) and/or the lack of tryptic cleavage sites, which would result in large peptide fragments that cannot be detected in crosslinked/MS analysis. To overcome the former limitation, we have mutated R155/236/245 to lysine residues in the context of both Ska1-MTBD and FL Ska1, and then tested these mutants in crosslinking/MS experiments (Supplementary Fig. S5c,d). Indeed, the R155/236/ $245 \mathrm{~K}$ mutant did reveal interactions between K155 and K245 of Ska1 with $\mathrm{H} 3$ of $\alpha$ - and $\beta$-tubulin, respectively (Fig. 4c).

Ska complex can bind MTs in multiple different orientations. The Lys clusters of Ska1 and the Asp/Glu clusters of tubulin monomers crosslinked in different ways with each other, suggesting the presence of multiple modes of Ska-MT interactions (Fig. 4c). For example, the K183/184 cluster (Ska1 $1_{\text {cluster1 }}$ ) crosslinked with both E110/E113/Y108 ( $\beta$-tub $\left._{\text {cluster1 }}\right)$ and E159/E160/ Y161 $\left(\beta\right.$-tub $\left._{\text {cluster } 2}\right)$ clusters of $\beta$-tubulin and the same could also be observed for the K203/206 cluster (Ska1 $1_{\text {cluster2 }}$; Fig. 4c). Moreover, the distance between Ska1 $1_{\text {cluster1 }}$ and Ska1 $1_{\text {cluster } 2}$ is the

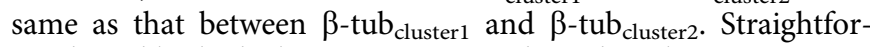
ward rigid body docking experiments show that Skal can interact with $\beta$-tubulin with Ska1 $1_{\text {cluster1 }}-S k a 1_{\text {cluster2 }}$, either facing

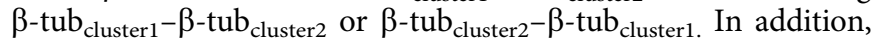
each Ska1 Lys cluster can interact individually with all tubulin Asp/Glu clusters. However, the assertion on the ability of the Ska complex to interact with MTs in multiple different orientations needs further validation.

Ska and Ndc80 complexes recognize different features of MTs. Structural characterizations of Ndc80-MT interactions revealed that the Ndc80 complex binds MTs by recognizing the dimeric interface of $\alpha$-and $\beta$-tubulins ${ }^{33}$. This mode of MT binding by the Ndc80 complex makes the interaction sensitive to the conformation of MT protofilaments. Indeed, the $\mathrm{Ndc80}$ complex preferentially binds straight MTs over curved MT protofilaments (vinblastine spirals; Fig. 5a and Supplementary Fig. S6b), in line with previous reports ${ }^{31,33}$. Our crosslinking/MS and cosedimentation data presented here show that the Ska complex, in contrast to the Ndc80 complex, interacts with MTs by recognizing the regions of tubulin monomers whose accessibility is not perturbed when MTs adopt different conformations. Thus, the Ska complex can bind straight and curved MT protofilaments indiscriminately using the same 
a
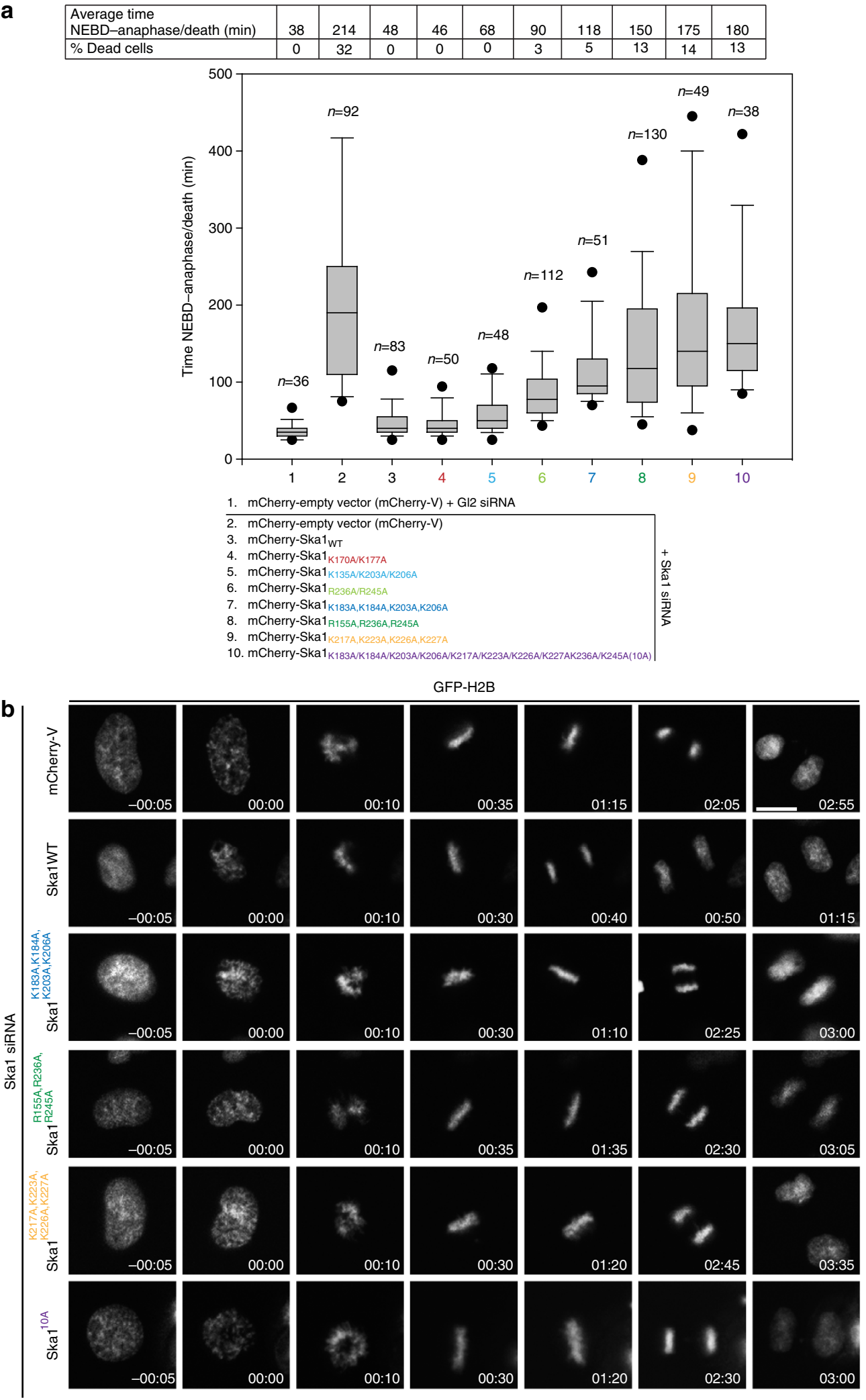

Figure 3 | MT recognition of Ska1 via its multipartite mode of MT binding is a functional requirement for the Ska complex. (a) Box-and-whisker plot showing the elapsed time ( $\mathrm{min}$ ) between nuclear envelope breakdown (NEBD) and anaphase onset/death for individual cells. The total number of cells (n) from two or more independent experiments is given above each box. Lower and upper whiskers represent 10th and 90th percentiles, respectively. Table summarizing information from the live cell experiments shown below regarding the average time in mitosis (from NEBD until anaphase onset/cell death) and the percentage of cells dying in mitosis. (b) Representative stills from time-lapse video-microscopy experiments illustrating mitotic progression of HeLa S3 cells stably expressing histone H2B-GFP. Scale bar, $10 \mu \mathrm{m}$. 
a

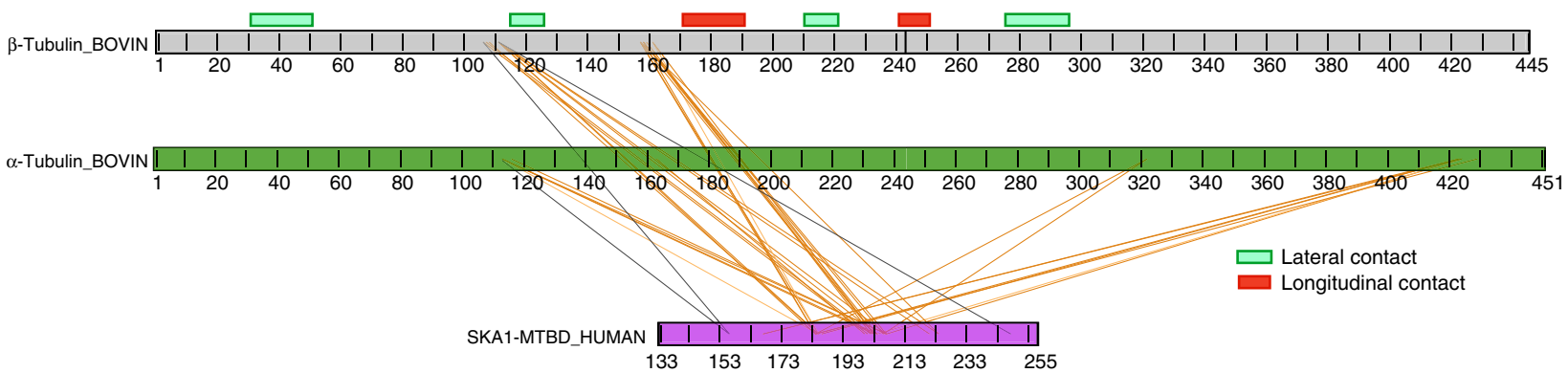

b

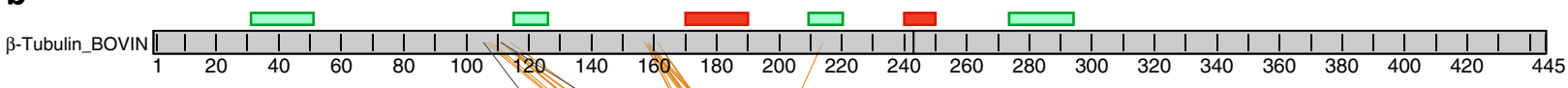

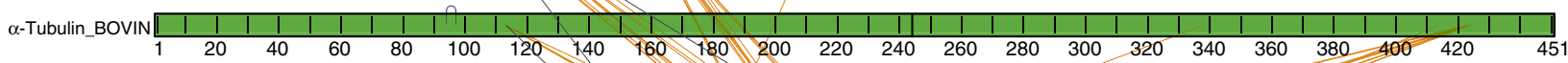
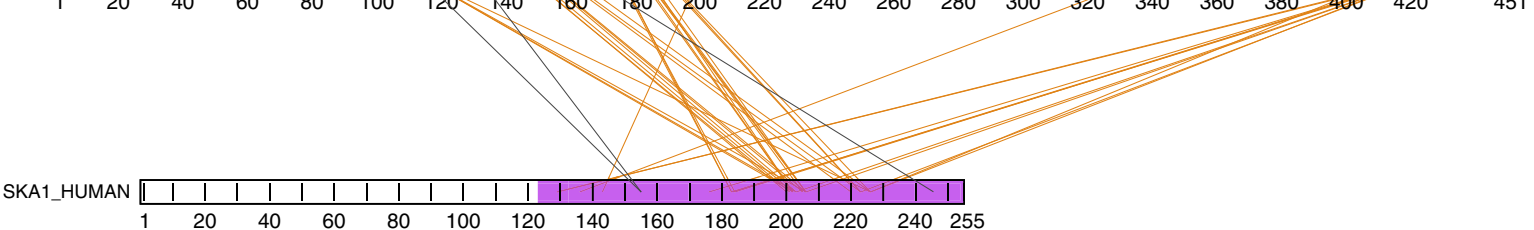

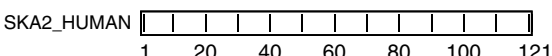

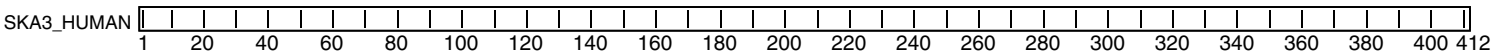

c

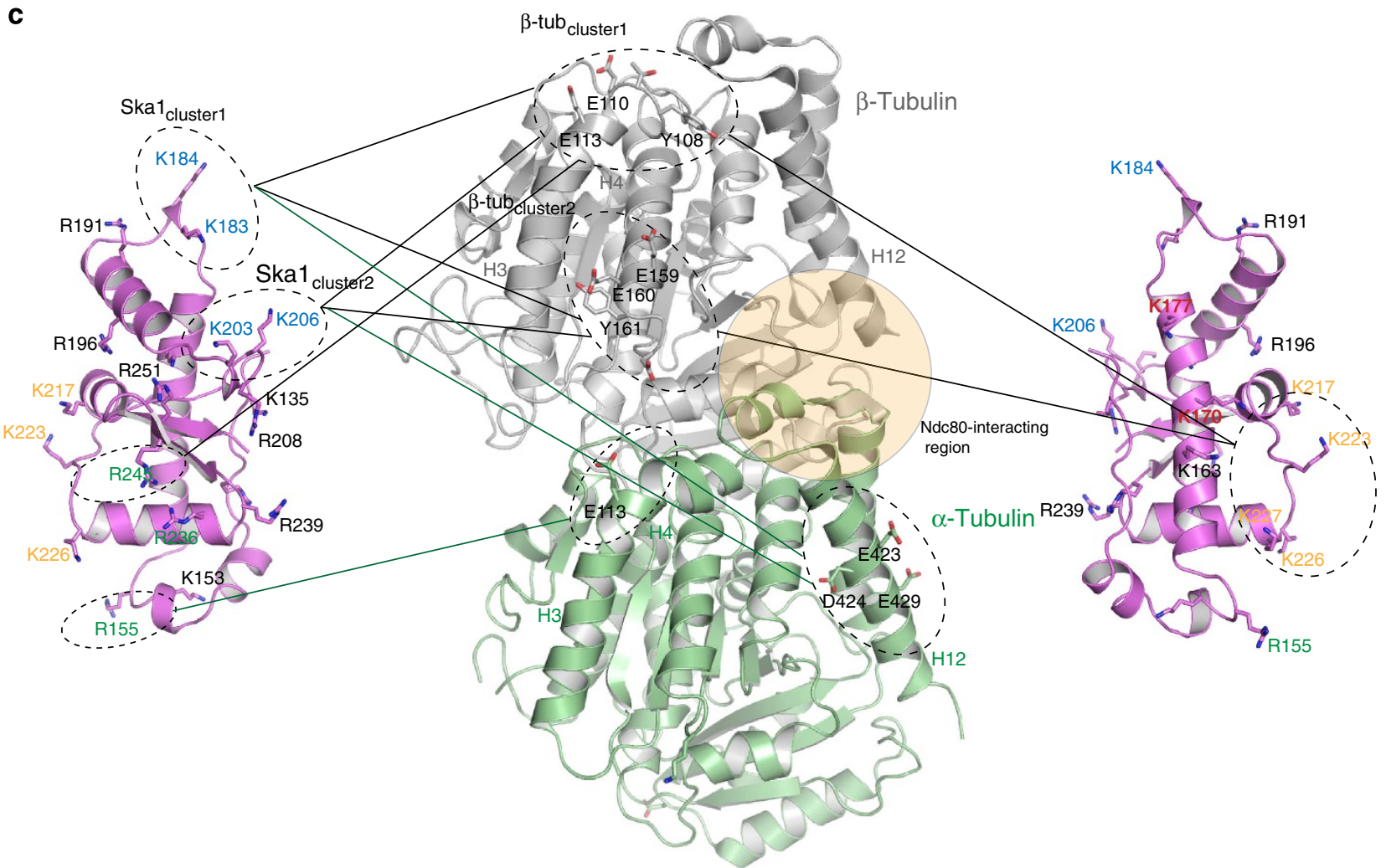

Figure 4 | Ska1 interacts with MTs by recognizing the globular regions of tubulin monomers in multiple orientations. (a,b) Linkage map showing the sequence position of all the crosslinked residue pairs between (a) Ska1-MTBD (b) Ska complex and $\alpha 1 B$ and $\beta 2 B$ tubulin, where $6 \mu$ M human Ska complex/ Ska1-MTBD was incubated with $10 \mu \mathrm{M}$ of MTs in the crosslinking reactions. Crosslinked products were resolved in SDS-PAGE followed by MS analysis. Red and green boxes above the $\beta$-tubulin show regions of tubulin involved in longitudinal and lateral contacts, respectively. Crosslinks observed between Ska1 R155/236/245K mutant and MTs are shown in grey. (c) Cartoon representation of tubulin dimer where residues involved in crosslinking with Ska1 residues are highlighted in stick representation. Grey and green lines denote crosslinks observed between K/R clusters of Ska1 and Glu/Asp/Tyr/Thr clusters of $\beta$-tubulin and $\alpha$-tubulin, respectively. The region where $\mathrm{Ndc} 80$ interacts with tubulin as reported in Alushin et al. ${ }^{33}$ is shown in yellow. Important Ska1 residues involved in MT binding are colour coded as in Figs 2 and 3. 
a
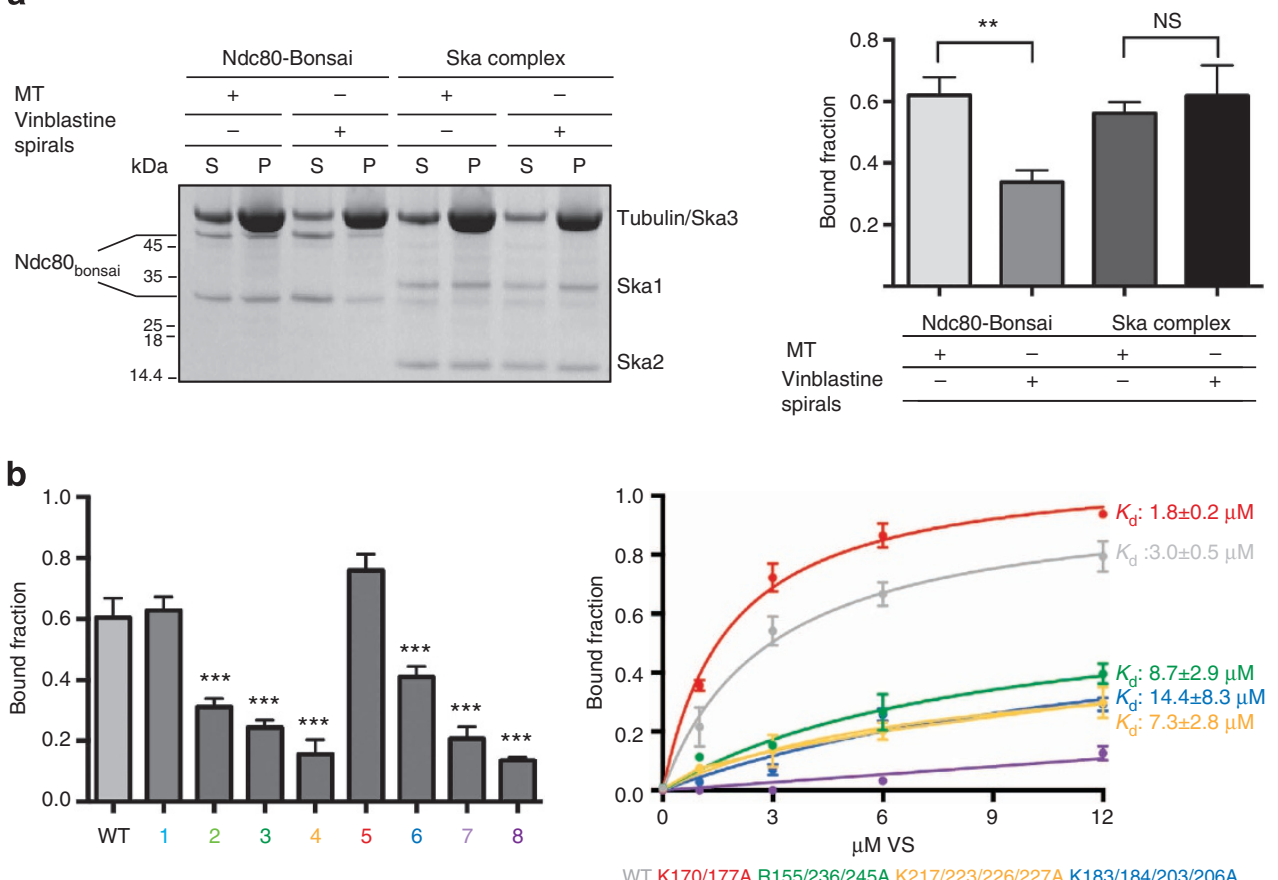

WT K170/177A R155/236/245A K217/223/226/227A K183/184/203/206A K183/184/203/206/217/223/226/227/R236/245A

C
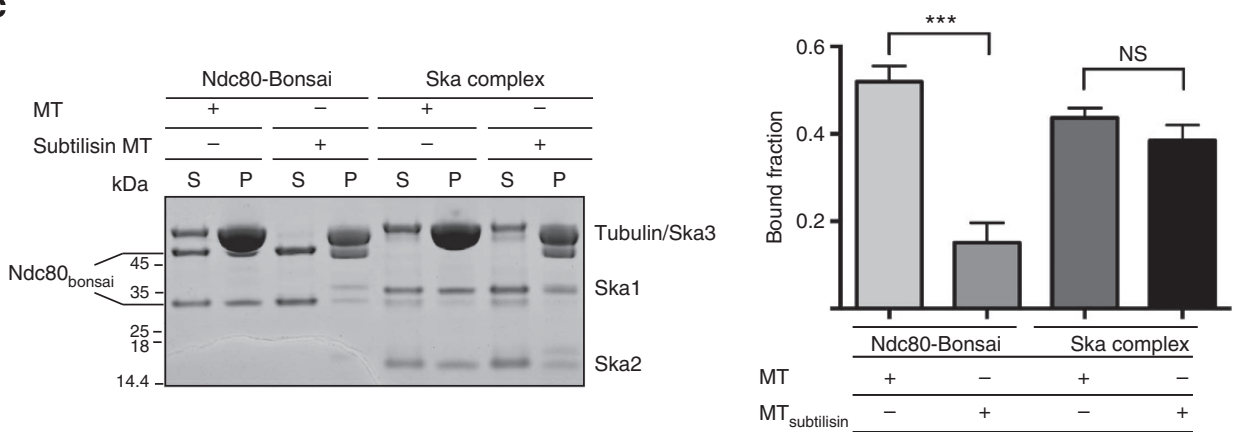

Figure 5 | Ska and Ndc80 complexes recognize different structural features of MTs. (a) Left, representative SDS-PAGE. Right, quantification of MT cosedimentation assays with $\mathrm{Ndc} 80$ and Ska complexes $(1 \mu \mathrm{M})$ binding to taxol-stabilized MTs or curved protofilaments induced by vinblastine ( $6 \mu \mathrm{M})$.

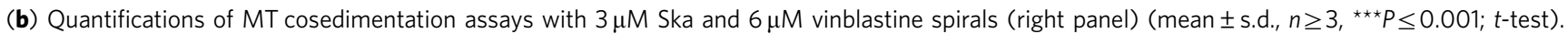
$K_{d}$ values were calculated using $1 \mu \mathrm{M}$ Ska and $0-12 \mu \mathrm{M}$ vinblastine spirals (left panel). (c) Left, representative SDS-PAGE. Right, quantification of MT

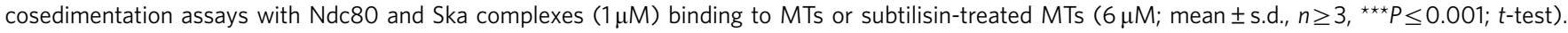
Uncropped scans of the gels are shown in Supplementary Fig. S8c.

contact sites (see $K_{\mathrm{d}}$ values for straight MTs in Fig. $2 \mathrm{~b}$ and corresponding values for vinblastine spirals in Fig. 5b). In future, it will be interesting to explore the possibility that the presence of the Ndc80 complex (or other Ska-binding partners) could induce some of the binding sites to discriminate between different MT structures.

MT-interacting proteins often interact with MTs by recognizing the acidic tails of tubulin, called 'E-hooks'. Biochemical and structural characterizations of Ndc80-MT interactions carried out in different laboratories have also highlighted the important contribution of the acidic tail of tubulin in the overall recognition of MTs by the Ndc80 complex ${ }^{13,33}$. Our crosslinking MS analysis does not provide insight into the possible role of acidic tubulin tails in Ska complex binding, owing to the technical reasons discussed in the previous section. However, as the Ska complex makes multiple contacts with the structured regions of tubulins, we hypothesized that the acidic tail of tubulin may not significantly contribute to the overall recognition of MTs. To evaluate this hypothesis, we tested the ability of the Ska complex to bind subtilisin-treated MTs (where E-hooks are removed by subtilisin treatment) in cosedimentation assays. As expected, the Ndc80 complex showed reduced binding to subtilisin MTs. Interestingly, the Ska complex did not show any noticeable reduction in its ability to bind subtilisin MTs, in line with the view that the critical contacts involve the structured regions of tubulin monomers rather than the acidic tails (Fig. $5 \mathrm{c}$ and Supplementary Fig. S6c).

Aurora B sites lie within the MT-binding K/R clusters. The role of Aurora B kinase in correcting erroneous KT-MT attachments by phosphorylating components of the KMN network, notably the Ndc80 complex and KNL1, is well established $11,34,43,44$. Recent work also showed that Aurora B negatively regulates the KT localization of the Ska complex, possibly influencing interactions with the KMN network ${ }^{30}$. As noted elsewhere ${ }^{31}$, all four Aurora B consensus sites (T157, S185, T205 and S242) are located within the Ska1-MTBD (Fig. 6a). Interestingly, two of 
a

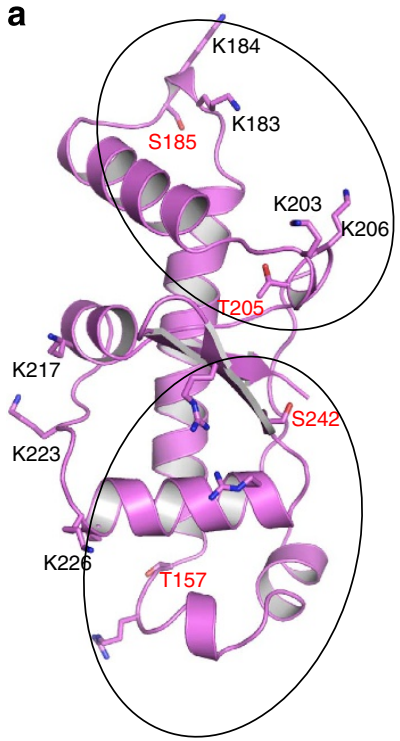

b

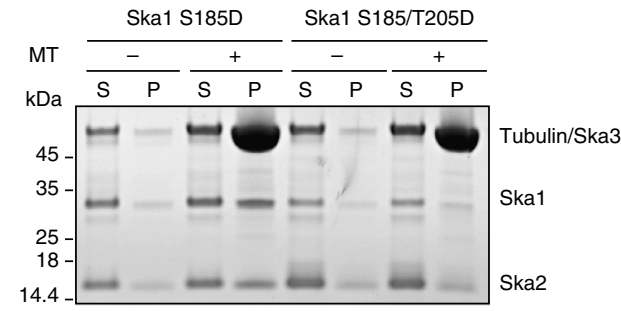

C
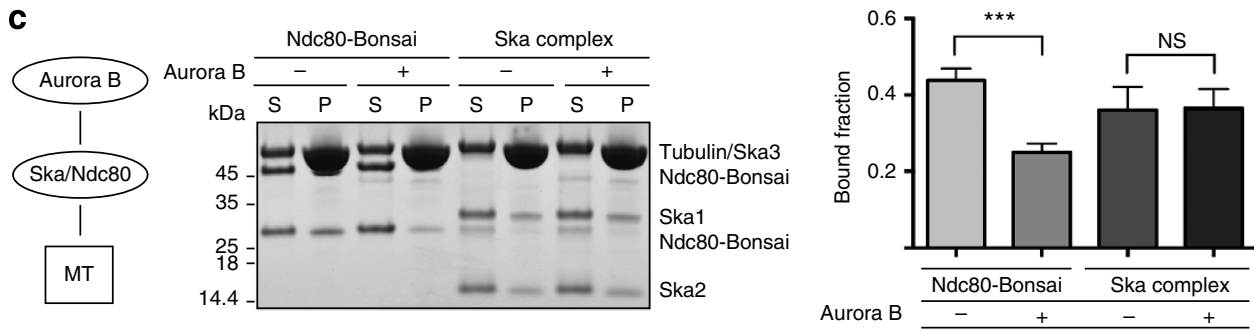

d
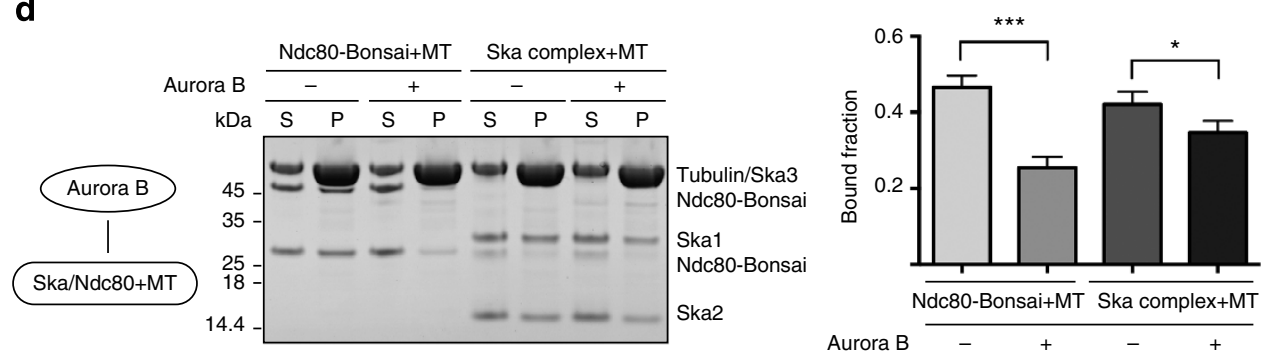

Figure 6 | Constitutive phosphorylation of S185/T205 perturbs MT binding of the Ska1-MTBD in vitro. (a) Cartoon representation of Ska1-MTBD, where consensus Aurora B phosphorylation sites are shown in stick representation (red). The phosphorylation sites of Aurora B are located within two of the three main clusters responsible for the MT-binding activity of Ska1 (circled). (b) Representative SDS-PAGE of the MT cosedimentation assays where phosphomimic mutants S185D and S185/T205D are tested. (c,d) Left, schematic representation of both protocols used for the treatment of Ska or Ndc80 complex with Aurora B. Middle, representative SDS-PAGE of the cosedimentation assays, where (c) the Ska/Ndc80 complex was incubated first with Aurora B kinase and subsequently with MTs. (d) MT-bound Ska/Ndc80 complex was incubated with Aurora B. Right, quantification of the results obtained (mean \pm s.d., $n \geq 3,{ }^{\star} P \leq 0.05,{ }^{\star \star \star} P \leq 0.001$; $t$-test). Uncropped scans of the gels are shown in Supplementary Fig. S8d.

these sites (S185 and T205) are located within the K/R cluster K183/184/203/206 that we have identified as being important for the MT-binding activity of the Ska complex (Fig. 6a). This suggested a direct involvement of S185 and T205 in the Aurora B-mediated phosphoregulation of Ska-MT interactions. To test this possibility, we made phosphomimicking mutants of Skal (S185D and S185/T205D) and tested them in MT-binding assays. Although S185D did not show any noticeable reduction in its ability to bind MTs, S185/T205D showed a drastic reduction (Fig. 6b and Supplementary Fig. S7). However, in line with our previous study ${ }^{30}$, pre-incubation of the Ska complex with Aurora $\mathrm{B}$ did not reduce its MT-binding ability, although the Ndc80 complex analysed for control responded as expected (Fig. 6c). To explain these apparently contradictory findings, we considered the possibility that efficient phosphorylation of Skal by Aurora B might require prior conformational rearrangements within the MTBD, which might occur either on MT binding or in the context of other KT-associated proteins. To test the former possibility, we first allowed the Ska complex to bind MTs before incubating the MT-bound Ska complex with Aurora B and evaluating the consequences in MT-binding assays. This experiment revealed a small but statistically significant reduction in the Ska-MT interaction in response to Aurora B (Fig. 6d). In line with the above model, normal mode analysis of the MD simulated structures of Ska1-MTBD shows the presence of an intrinsic flexibility associated with the structural element that harbours this MT-binding site and Aurora B consensus sites (Supplementary Movie 10). The other Aurora B sites, S157 and 
S242, are also close to the R cluster R155/236/245, but insertion of phosphomimicking mutations did not show any influence on MT binding (Supplementary Fig. S7). It is interesting that combination of a phosphomimic mutation at S242D did not abolish MT binding in combination with T157D (this study), although it has been previously shown that combined mutations at S242D and S185D strongly reduced MT binding. This observation, together with our observation that the S185D/ T205D combination also drastically diminishes MT binding, demonstrates that Aurora B phosphorylation of Ska1-MTBD can negatively regulate Ska-MT interactions via multiple phosphorylation events.

\section{Discussion}

MT-binding activity at the $\mathrm{KT}$ is a prime requirement for driving accurate chromosome segregation. Although the Ndc80 complex is considered to be the major contributor to MT binding by the $\mathrm{KT}$, other factors such as the Dam1 complex in budding yeast and the Ska complex in vertebrates are required to efficiently couple MT binding at the KT with chromosome segregation ${ }^{5}$. The ability of the Dam1 complex to form rings around MTs suggested that Dam1-like proteins might work as force couplers that harness the force associated with MT depolymerization with chromosome movement $t^{5,45}$. However, the proposed functional homologue of Dam1 in humans, the Ska complex, forms a flexible W-shaped structure of triple helical bundles with a maximum interatomic distance of $35 \mathrm{~nm}$ (ref. 36). With MTBDs symmetrically positioned at both ends of the dimer, the Ska complex thus appears to exert its function via a different mechanism.

Here we have used biochemical and high-resolution structural analysis to show that the human Ska complex interacts with MTs through the C-terminal domain of Ska1, which forms a variant form of winged-helix domain. This domain has previously been seen as a DNA-binding module in transcription regulators but also as a protein interaction module in a small number of proteins with diverse functions ${ }^{38,41,42}$. The Ska complex provides the first instance where this module is used as a MTBD. However, considering that, first, both DNA molecules and MTs are often recognized through electrostatic interactions exploiting the negatively charged nature of these molecules, and, second, that MT-based diffusional motility and DNA-based diffusion shows striking similarities with comparable diffusion coefficients ${ }^{46}$, the use of a winged-helix domain for the Ska1-MTBD may not be surprising. It will be interesting to see whether the Dam1 complex also possesses winged-helix domains in its MT-binding components.

Structural comparisons of human and C. elegans Ska1MTBDs $^{31}$ show conformational variations in the regions that we demonstrate here to be critical for MT binding. This adds to species-specific differences in the overall composition and architecture of the Ska complexes, in that Ska1 and Ska3 associate in a 2:1 complex in C. elegans ${ }^{31}$, whereas the human Ska complex is made of Ska1, Ska2 and Ska3 in a 2:2:2 ratio $^{36}$. It is tempting to suggest that the attachment of chromosomes to spindle MTs in vertebrates versus nematodes may exhibit different dynamic properties. In organisms with 'holocentric' chromosomes, such as C. elegans, MTs are attached at multiple sites along the chromosome arms, resulting in chromosomes with no apparent dynamic oscillations at metaphase. In contrast, in 'monocentric' mammalian cells, chromosomes are attached to MTs at a discrete site and metaphase-aligned chromosomes show pronounced dynamics ${ }^{47}$.

Using MT-binding assays in combination with siRNA-based rescue assays, we demonstrate here the involvement of at least three tubulin contact sites within Ska1-MTBD for MT recognition.
Remarkably, the tubulin contact sites are dispersed across the surface of the Ska1-MTBD and distances between different contact sites range between 15 and $30 \AA$. Disruption of even one of the tubulin contact sites is enough to perturb normal mitotic progression, suggesting the requirement of all contact sites for efficient function. Crosslinking/MS analysis revealed important molecular details of these Ska-MT interactions, in particular the novel ability of the Ska-MTBD to bind to tubulin monomers. This data together with those from quantitative MT cosedimentation assays demonstrated that tubulin contacts of the Ska1-MTBD can bind straight and curved MTs with no apparent preference.

Identifying and characterizing the unique properties of the $\mathrm{Ndc} 80$ and Ska complexes is crucial for understanding how these complexes complement each other in providing an integrated interface for efficient MT binding and MT-driven motility. The $\mathrm{Ndc} 80$ complex binds MTs through the interaction of the Ndc80$\mathrm{CH}$ (Calponin Homology) domain (called the 'toe') at the tubulin dimeric interface (called the 'toe print') in a way that favours interactions with the straight conformation of MT protofilaments ${ }^{33}$. Our results show that the Ska complex interacts with the structured regions of tubulin monomers, mainly at helices $\mathrm{H} 3$ and $\mathrm{H} 4$ of $\beta$-tubulin and $\mathrm{H} 12$ of $\alpha$-tubulin, whose accessibility is not perturbed on MTs assuming different conformations. This feature gives the Ska complex the ability to bind both straight and curved protofilaments with equal efficiency (Fig. 7). One of the sites (Glu110, 156 and 162 in H3) through which $\beta$-tubulin makes contact with the Ska1-MTBD is particularly intriguing, as this site is close to the GTP-binding site and also near the regions involved in lateral contacts between adjacent MT protofilaments. Furthermore, this site is recognized by EB1 and has been suggested to be important for EBl's end-tracking activity and for stabilizing growing $\mathrm{MTs}^{48}$. In this context, it would be interesting to know whether the Ska complex can also influence MT dynamics.

One of the intriguing observations made in our study concerns the ability of the Ska complex to bind MTs in multiple different orientations. This combines with the fact that the Ska1-MTBDs are loosely connected to the $\mathrm{W}$-shaped triple helical structure through a 40 amino acid loop, thus providing additional flexibility that may be important to allow the KTs to track disassembling MTs (Fig. 7). This is in stark contrast to the $\mathrm{Ndc} 80$ complex, where $\mathrm{CH}$ domains of $\mathrm{Ndc} 80$ are connected to a rather rigid helical bundle ${ }^{13,33}$. We further envisage that the presence of this loop in Ska1 is likely to be critical for efficient MT tracking by the Ska complex and possibly for mediating protein-protein interactions with other KT components. In support of this view, we found that deletion of the Skal loop delays mitotic progression of dividing cells.

In summary, our results indicate that the function of the Ska complex is conferred by its ability to interact with regions of tubulin monomers whose accessibility is not affected by different MT structures. These interactions involve multipartite binding sites and allow MT binding in multiple orientations (Fig. 7). Future structural and functional studies on whether and how Ska-MT interactions are modulated by the presence of other MT-binding proteins, notably the Ndc80 complex, will advance our understanding of the molecular underpinnings of KT-MT attachments and chromosome segregation.

\section{Methods}

Expression and purification of recombinant proteins. Ska1 $1_{133-255}$ was cloned into a pEC-K-3C-His-GST vector as an N-terminally His-GST-tagged protein with a 3C-cleavage site. Ska1, Ska2 and Ska3 V58I were cloned individually in a pEC-S-CDF-His, pEC-A-HT-His GST and pEC-K-HT-His vectors, respectively, with TEV cleavage sites. Skal mutants were generated following the Quikchange site-directed mutagenesis method (Stratagene; primer details are given in Supplementary Table S3). To express the Ska complex containing Ska1 $\Delta$ loop, 


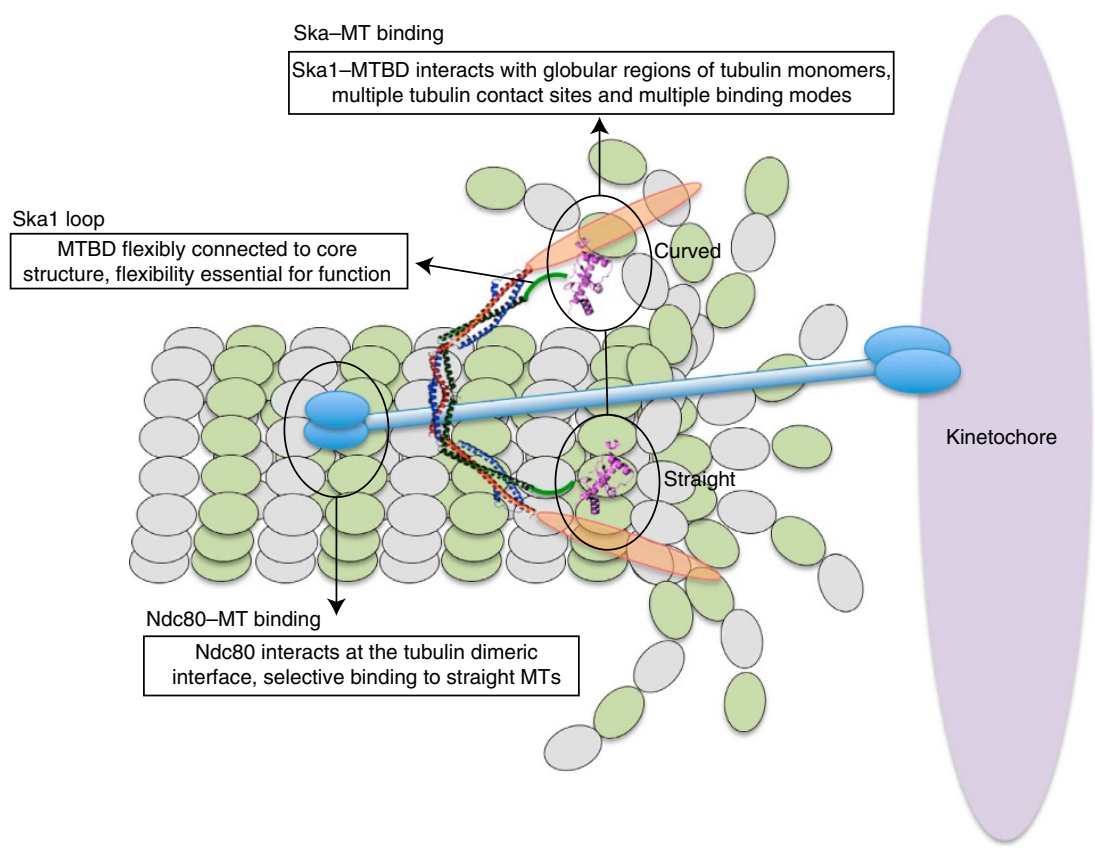

Figure 7 | Schematic model. Schematic model summarizing the mode of MT binding of Ska1 and its implications for maintaining stable KT-MT attachments.

Ska1 $\Delta$ loop(GSSG) and phosphomimic mutants, Quikchange site-directed mutagenesis was performed on a polycistronic vector (primer details are given in Supplementary Table S3), pETMCN (gift from C. Romier, IGBMC, Strasbourg) containing GST-Ska2 (3C-cleavable), untagged Ska1 and Ska3. All protein complexes were expressed in Escherichia coli strain BL21 Gold, either using the polycistronic constructs or by cotransforming all the three plasmids containing individual Ska components. Cultures were induced overnight at $18^{\circ} \mathrm{C}$ and purified using a similar protocol. Cells were lysed in a buffer containing $20 \mathrm{mM}$ Tris, $\mathrm{pH} 8$, $500 \mathrm{mM} \mathrm{NaCl}$ and $5 \mathrm{mM}$ dithiothreitol (DTT). The protein complexes were purified by affinity chromatography in batch mode using glutathione sepharose (GE Healthcare) beads. Protein-bound beads were washed with $20 \mathrm{mM}$ Tris, $\mathrm{pH} 8$, $500 \mathrm{mM} \mathrm{NaCl}$ and $5 \mathrm{mM}$ DTT, followed by $20 \mathrm{mM}$ Tris, $\mathrm{pH} 8,1 \mathrm{M} \mathrm{NaCl}, 50 \mathrm{mM}$ $\mathrm{KCl}, 10 \mathrm{mM} \mathrm{MgCl} 2,2 \mathrm{mM}$ ATP and $5 \mathrm{mM}$ DTT, then finally with $20 \mathrm{mM}$ Tris, $\mathrm{pH} 8$, $100 \mathrm{mM} \mathrm{NaCl}$ and $5 \mathrm{mM}$ DTT. Proteins with 3C-cleavage sites were cleaved, while the proteins were still bound to the beads. TEV cleavable proteins were eluted with $50 \mathrm{mM}$ glutathione, $20 \mathrm{mM}$ Tris, $\mathrm{pH} 8,100 \mathrm{mM} \mathrm{NaCl}$ and $5 \mathrm{mM}$ DTT, and the tags were removed in solution overnight. Subsequently, proteins/protein complexes were purified by size-exclusion chromatography in $20 \mathrm{mM}$ Tris, $\mathrm{pH} 8,100 \mathrm{mM} \mathrm{NaCl}$ and $5 \mathrm{mM}$ DTT (Superose 6, GE Healthcare). Ndc80 Bonsai (kindly gifted by Andrea Musacchio) was expressed in E. coli BL21 (DE3) ${ }^{13}$. Cells were lysed in lysis buffer containing $50 \mathrm{mM}$ Tris, $\mathrm{pH}$ 7.6, $300 \mathrm{mM} \mathrm{NaCl}, 1 \mathrm{mM}$ DTT and $1 \mathrm{mM}$ EDTA. Cleared lysate was incubated with glutathione sepharose beads. After $3 \mathrm{~h}$ incubation at $4{ }^{\circ} \mathrm{C}$, beads were washed with $50 \mathrm{mM}$ Tris, $\mathrm{pH} 7.6,150 \mathrm{mM} \mathrm{NaCl}, 1 \mathrm{mM}$ DTT and $1 \mathrm{mM}$ EDTA, and cleaved with $3 \mathrm{C}$ protease for $16 \mathrm{~h}$ at $4{ }^{\circ} \mathrm{C}$. Concentrated protein was loaded onto a Superose 6 size-exclusion chromatography column (GE Healthcare) equilibrated with $20 \mathrm{mM}$ Tris, $\mathrm{pH}$ 7.6, $100 \mathrm{mM} \mathrm{NaCl}, 1 \mathrm{mM}$ DTT and $10 \%$ glycerol.

Crystallization and data collection. Crystallization trials were performed using a nanolitre crystallization robot at the Edinburgh Protein Production Facility. Crystals of form I $\left(\mathrm{C} 222_{1}\right)$ were grown by vapour diffusion method using Morpheus condition C2, mother liquor containing $0.1 \mathrm{M}$ Imidazole-MES buffer, $0.09 \mathrm{M}$ NPS mix $\left(\mathrm{NaNO}_{3}, \mathrm{Na}_{2} \mathrm{HPO}_{4},\left(\mathrm{NH}_{4}\right)_{2} \mathrm{SO}_{4}\right)$ and $30 \%$ EDO_P8K (ethylene glycol; PEG $8 \mathrm{~K}$ ) (with $1 \mu \mathrm{l}$ of $15-20 \mathrm{mg} \mathrm{ml}^{-1}$ protein sample mixed with $1 \mu \mathrm{l}$ of mother liquor). Crystals of form II $\left(\mathrm{P}_{2}\right)$ were grown in mother liquor containing $24 \%$ (w/v) PEG 1,500 and $20 \%$ glycerol. As the crystallization conditions were suitable to act as cryoprotectants, crystals were directly flash frozen in liquid nitrogen. The crystals diffracted to about $2 \AA$ resolution at the MX beamlines of the Diamond Light Source (Table 1).

Crystal structure solution and refinement. The structure of the Ska1-MTBD (form I) was determined by the SAD method, using the data collected at the selenium (SE) edge (0.97 $\AA$ ). Data was processed using XDS and scaled with SCALA of CCP4 (ref. 49). SAD phasing and the calculation of the initial map were performed using phenix.autosol from the PHENIX suite of programmes ${ }^{50}$. The model was built by iterative rounds of manual building with COOT $^{51}$ and refinement using phenix.refine of PHENIX suite of programmes ${ }^{50}$. The structure of the Ska1-MTBD from $\mathrm{P}_{2}$ space group was determined using molecular replacement method using PHENIX suite of programmes ${ }^{50}$. Data collection, phasing and refinement statistics are shown in Table 1.

Molecular dynamics simulations. For the MDS, chain B of crystal form I (C222 $)$ and model 1 of NMR structure of the C. elegans (pdb: 2LYC) was used. MD studies were carried out using the AMBER12 (ref. 52) package. The missing atoms were built using standard geometries as implemented in AMBER. Each system was solvated with a box of TIP3P water molecules such that the boundary of the box was at least $10 \AA$ from any protein atom. The net positive charges in the system were balanced by adding chloride ions. The force field ff12SB was used for intermolecular interactions. The particle mesh Ewald method was used for treating the long-range electrostatics. All bonds involving hydrogen were constrained by SHAKE. An integration time step of $2 \mathrm{fs}$ was used for propagating the dynamics. Each system was initially minimized for 3,000 steps to remove any unfavourable interactions between the protein and the solvent, followed by heating to $300 \mathrm{~K}$ over $30 \mathrm{ps}$ under normal pressure/temperature conditions. Subsequently, each system was simulated for $50 \mathrm{~ns}$ at constant temperature $(300 \mathrm{~K})$ and pressure $(1 \mathrm{~atm})$, and the structures were stored every $10 \mathrm{ps}$ for analysis. Analysis was carried out using $\mathrm{VMD}^{53}$.

MT cosedimentation assays. Tubulin was purchased from Cytoskeleton Inc. and MTs were polymerized according to manufacturer's instructions. To generate vinblastine spirals, tubulin was diluted to $3 \mathrm{mg} \mathrm{ml}^{-1}$ in $80 \mathrm{mM}$ PIPES, pH 6.8 , $1 \mathrm{mM}$ EGTA, $1 \mathrm{mM} \mathrm{MgCl} 2,1 \mathrm{mM}$ DTT and 5\% sucrose supplemented with $3 \mathrm{mM}$ vinblastine sulphate (Sigma-Aldrich) at room temperature for $2 \mathrm{~h}$. Subtilisin-treated MTs were obtained after incubation of $6 \mu \mathrm{M}$ taxol-stabilized MTs for $45 \mathrm{~min}$ at $30^{\circ} \mathrm{C}$ with $100 \mu \mathrm{g} \mathrm{ml}^{-1}$ subtilisin A (Sigma-Aldrich) following a previously reported procedure ${ }^{13}$. The reaction was stopped with $10 \mathrm{mM}$ phenylmethyl sulphonyl fluoride and the digested MTs pelleted $(434,400 \mathrm{~g}$, TLA $100.3,10 \mathrm{~min}$, $\left.25^{\circ} \mathrm{C}\right)$ and resuspended in the original volume of general tubulin buffer $(80 \mathrm{mM}$ PIPES, pH 6.9, $2 \mathrm{mM} \mathrm{MgCl}_{2}$ and $0.5 \mathrm{mM}$ EGTA).

For MT-pelleting assays, taxol-stabilized MTs, vinblastine spirals or subtilisintreated MTs $(0-12 \mu \mathrm{M}$ tubulin dimer as stated in each experiment) were incubated at room temperature for $10 \mathrm{~min}$ with 1 or $3 \mu \mathrm{M}$ protein (Ska or Ndc80 bonsai) in a $50-\mu \mathrm{l}$ reaction volume in BRB80 buffer $(80 \mathrm{mM}$ PIPES, $\mathrm{pH} 6.9,1 \mathrm{mM}$ EGTA and $1 \mathrm{mM} \mathrm{MgCl}$ ) with $100 \mathrm{mM} \mathrm{NaCl}$ and $4 \mathrm{mM}$ DTT in the presence of $20 \mu \mathrm{M}$ taxol or $3 \mathrm{mM}$ vinblastine. The reaction was then layered onto a $250-\mu \mathrm{l}$ glycerol cushion buffer (BRB80, 50\% glycerol, 4 mM DTT for taxol-stabilized MTs and BRB80, 30\% glycerol, $4 \mathrm{mM}$ DTT for vinblastine spirals with the appropriate drug) and ultracentrifuged for $10 \mathrm{~min}$ at $434,400 \mathrm{~g}$ in a Beckman TLA 100.3 rotor at $25^{\circ} \mathrm{C}$. Pellets and supernatants were analysed by SDS-PAGE. Gels were stained with Coomassie Blue gel staining and quantification was performed with Image ${ }^{54}$. Normalized binding data were obtained by dividing the values of the pellet fraction 
by the sum of pellet and supernatant. Fitting analysis and $K_{\mathrm{d}}$ calculations were carried out using GraphPad Prism, version 6.0 (GraphPad Software, Inc).

For the Aurora B assay, we incubated $3 \mu \mathrm{M}$ Ska complex or $\mathrm{Ndc} 80$ bonsai with $10 \mathrm{mM}$ ATP, $20 \mathrm{mM} \mathrm{MgSO}_{4}$ and $900 \mathrm{nM}$ Aurora B for $30 \mathrm{~min}$ at $30^{\circ} \mathrm{C}$. Taxolstabilized MTs ( $6 \mu \mathrm{M}$ tubulin dimer) were then added for $10 \mathrm{~min}$ at room temperature and cosedimentation assays were performed as described above. In the second batch of experiments, we first incubated the protein (Ska complex or Ndc80 bonsai) with the taxol-stabilized MTs for $10 \mathrm{~min}$ and then after we incubated for 30 min at $30^{\circ} \mathrm{C}$ with Aurora B.

Chemical crosslinking and MS analysis. Crosslinking experiments were carried out using a zero-length crosslinking agent, EDC (Thermo Fisher Scientific) in the presence of $N$-hydroxysulphosuccinimide (Thermo Fisher Scientific). Ska complex $(6 \mu \mathrm{M})$ and $10 \mu \mathrm{M}$ MTs were incubated with $10 \mu \mathrm{g}$ EDC and $22 \mu \mathrm{g} N$-hydroxysulphosuccinimide in a final volume of $20 \mu \mathrm{l}$. The reaction mixture was incubated for $90 \mathrm{~min}$ at $25^{\circ} \mathrm{C}$ and was quenched by adding Tris- $\mathrm{Cl}$ to a final concentration of $100 \mathrm{mM}$. The reactions were resolved by SDS-PAGE (4-12\% Bis-Tris NuPAGE, Invitrogen) gel separation and stained using Instant Blue (Expedeon). The bands corresponding to crosslinked complexes were excised and the proteins therein were reduced using $10 \mathrm{mM}$ DTT for $30 \mathrm{~min}$ at room temperature, alkylated with $55 \mathrm{mM}$ iodoacetamide for $20 \mathrm{~min}$ in the dark at room temperature and digested using $13 \mathrm{ng} \mathrm{l}^{-1}$ trypsin (sequencing grade; Promega) overnight at $37^{\circ} \mathrm{C}$ (ref. 14). The digested peptides were desalted using C18-StageTips $^{55}$ and analysed on a LTQ Orbitrap Velos mass spectrometer (Thermo Fisher Scientific $)^{56-58}$. An analytical column with a spray emitter $(75 \mu \mathrm{m}$ inner diameter, $8 \mu \mathrm{m}$ opening, $250 \mathrm{mM}$ length; New Objectives) that was packed with C18 material (ReproSil-Pur C18-AQ $3 \mu \mathrm{m}$; Dr Maisch GmbH, Ammerbuch-Entringen, Germany) using an air pressure pump (Proxeon Biosystems). Mobile phase A consisted of water with $0.1 \%$ formic acid. Mobile phase B consisted of $80 \%$ acetonitrile with $0.1 \%$ formic acid. Peptides were loaded onto the column with $1 \%$ $\mathrm{B}$ at $600 \mathrm{nl} \mathrm{min}{ }^{-1}$ flow rate and eluted at $300 \mathrm{nl} \mathrm{min}^{-1}$ flow rate, with a linear gradient increased from 5 to $35 \%$ acetonitrile in $0.1 \%$ formic acid in $150 \mathrm{~min}$ to elute peptides. Peptides were analysed using a high/high strategy; both MS spectra and MS2 spectra were acquired in the Orbitrap ${ }^{56}$. Mass spectra were recorded at 100,000 resolution. The eight highest intensity peaks with a charge state of three or higher were selected in each cycle for ion-trap fragmentation. The fragments were produced using collision-induced dissociation with $35 \%$ normalized collision energy and detected by the Orbitrap at 7,500 resolution. Dynamic exclusion was set to $30 \mathrm{~s}$ and repeat count was 1 . The data were processed, generating peak lists by MaxQuant ${ }^{59}$ and matching crosslinked peptides to spectra using in-house developed Xi software.

Cell culture and siRNA depletion. HeLa S3, and HeLa S3 cells expressing histone $\mathrm{H} 2 \mathrm{~B}-\mathrm{GFP}^{60}$, were routinely maintained in DMEM (Invitrogen) supplemented with $10 \%$ fetal bovine serum and penicillin/streptomycin $\left(100 \mathrm{IU} \mathrm{ml}^{-1}\right.$ and $100 \mathrm{mg} \mathrm{ml}^{-1}$, respectively; Gibco). For synchronization studies, cells were arrested for $20 \mathrm{~h}$ with $2 \mathrm{mM}$ thymidine, followed by a release into fresh medium for $6-8 \mathrm{~h}$ and a second thymidine block of $16 \mathrm{~h}$ and release for $10 \mathrm{~h}$ before fixation or visualization ${ }^{30,36}$. All Ska constructs were generated in the pcDNA3.1 plasmid (Invitrogen), driven by the cytomegalovirus promoter, and modified to carry an $\mathrm{N}$-terminal triple-Myc tag or a single mCherry tag. For rescue experiments, Ska1 siRNA-resistant constructs were used. Plasmid transfections were performed using TransIT-LT1 reagent (Mirus Bio Corporation) according to the manufacturer's instructions. siRNA duplexes were transfected using Oligofectamine (Invitrogen) according to the manufacturer's instructions. The sequences of the Ska1 (ref. 27) and control GL2 duplexes ${ }^{61}$ are: $5^{\prime}$-CCCGCTTAACCTATAATCAAA- $3^{\prime}$ and $5^{\prime}$-AACGTACGCGGAATACTTCGA- $3^{\prime}$, respectively. For western blotting, a rabbit anti-Ska1 antibody $(1: 1,000)$ and a mouse anti- $\alpha$-Tubulin antibody (DM1 $\alpha$ 1:2,000; Sigma) were used ${ }^{27}$.

Immunofluorescence and time-lapse microscopy. Cells grown on coverslips were fixed and permeabilized simultaneously in PTEMF buffer ( $0.2 \%$ Triton X-100, $20 \mathrm{mM}$ PIPES, pH 6.8, $1 \mathrm{mM} \mathrm{MgCl} 2,10 \mathrm{mM}$ EGTA and $4 \%$ formaldehyde). Cells were stained with mouse anti-myc 9E10 monoclonal antibody (culture supernatant, $1: 2$ ) and human CREST autoimmune serum (1:2,000; Immunovision). DNA was visualized with $4^{\prime}, 6$-diamidino-2-phenylindole $\left(2 \mu \mathrm{g} \mathrm{ml}^{-1}\right)$. All primary antibodies were detected with Cy2/Cy3-conjugated donkey antibodies (Dianova). A Deltavision microscope (Applied Precision) was used for immunofluorescence processing and image acquisition ${ }^{60}$. For time-lapse microscopy, all treatments within a single experiment were performed simultaneously. Cells were imaged using a Nikon ECLIPSE Ti microscope equipped with a CoolLED pE-1 excitation system and a $20 \times / 0.75$ air Plan Apo objective (Nikon). During imaging, the atmosphere was maintained at a temperature of $37^{\circ} \mathrm{C}$, humidity 60 and $5 \% \mathrm{CO}_{2}$. Images were captured at 5-min intervals for $22 \mathrm{~h}$ at multiple positions. Green fluorescent protein and mCherry fluorescence images were acquired at each time point with $30 \mathrm{~ms}$ and $60 \mathrm{~ms}$ exposure times, respectively. mCherry fluorescence was imaged only every five time point to monitor transfected cells. MetaMorph 7.7 software (MDS Analytical Technologies) was used to collect and process data.

\section{References}

1. Gascoigne, K. E. \& Cheeseman, I. M. Kinetochore assembly: if you build it, they will come. Curr. Opin. Cell Biol. 23, 102-108 (2011).

2. Santaguida, S. \& Musacchio, A. The life and miracles of kinetochores. EMBO J. 28, 2511-2531 (2009).

3. Joglekar, A. P., Bloom, K. S. \& Salmon, E. D. Mechanisms of force generation by end-on kinetochore-microtubule attachments. Curr. Opin. Cell Biol. 22, 57-67 (2010).

4. Foley, E. A. \& Kapoor, T. M. Microtubule attachment and spindle assembly checkpoint signalling at the kinetochore. Nat. Rev. Mol. Cell Biol. 14, 25-37 (2013).

5. DeLuca, J. G. \& Musacchio, A. Structural organization of the kinetochoremicrotubule interface. Curr. Opin. Cell Biol. 24, 48-56 (2012).

6. Tanaka, K. Regulatory mechanisms of kinetochore-microtubule interaction in mitosis. Cell. Mol. Life Sci. 70, 559-579 (2013).

7. Koshland, D. E., Mitchison, T. J. \& Kirschner, M. W. Polewards chromosome movement driven by microtubule depolymerization in vitro. Nature 331, 499-504 (1988).

8. Grishchuk, E. L. \& McIntosh, J. R. Microtubule depolymerization can drive poleward chromosome motion in fission yeast. EMBO J. 25, 4888-4896 (2006).

9. Grishchuk, E. L., Molodtsov, M. I., Ataullakhanov, F. I. \& McIntosh, J. R. Force production by disassembling microtubules. Nature 438, 384-388 (2005).

10. Tanaka, K., Kitamura, E., Kitamura, Y. \& Tanaka, T. U. Molecular mechanisms of microtubule-dependent kinetochore transport toward spindle poles. J. Cell Biol. 178, 269-281 (2007).

11. Cheeseman, I. M., Chappie, J. S., Wilson-Kubalek, E. M. \& Desai, A. The conserved KMN network constitutes the core microtubule-binding site of the kinetochore. Cell 127, 983-997 (2006).

12. Espeut, J., Cheerambathur, D. K., Krenning, L., Oegema, K. \& Desai, A. Microtubule binding by KNL-1 contributes to spindle checkpoint silencing at the kinetochore. J. Cell Biol. 196, 469-482 (2012).

13. Ciferri, C. et al. Implications for kinetochore-microtubule attachment from the structure of an engineered Ndc80 complex. Cell 133, 427-439 (2008).

14. Maiolica, A. et al. Structural analysis of multiprotein complexes by crosslinking, mass spectrometry, and database searching. Mol. Cell Proteomics 6, 2200-2211 (2007).

15. Malvezzi, F. et al. A structural basis for kinetochore recruitment of the $\mathrm{Ndc80}$ complex via two distinct centromere receptors. EMBO J. 32, 409-423 (2013).

16. Lampert, F., Mieck, C., Alushin, G. M., Nogales, E. \& Westermann, S. Molecular requirements for the formation of a kinetochore-microtubule interface by Daml and Ndc80 complexes. J. Cell Biol. 200, 21-30 (2013).

17. Umbreit, N. T. et al. The Ndc80 kinetochore complex directly modulates microtubule dynamics. Proc. Natl Acad. Sci. USA 109, 16113-16118 (2012).

18. Powers, A. F. et al. The Ndc80 kinetochore complex forms load-bearing attachments to dynamic microtubule tips via biased diffusion. Cell 136, 865-875 (2009)

19. Miranda, J. J., De Wulf, P., Sorger, P. K. \& Harrison, S. C. The yeast DASH complex forms closed rings on microtubules. Nat. Struct. Mol. Biol. 12, 138-143 (2005)

20. Westermann, S. et al. The Dam1 kinetochore ring complex moves processively on depolymerizing microtubule ends. Nature 440, 565-569 (2006).

21. Lampert, F., Hornung, P. \& Westermann, S. The Dam1 complex confers microtubule plus end-tracking activity to the $\mathrm{Ndc} 80$ kinetochore complex. J. Cell Biol. 189, 641-649 (2010).

22. Tien, J. F. et al. Cooperation of the Dam1 and Ndc80 kinetochore complexes enhances microtubule coupling and is regulated by aurora B. J. Cell Biol. 189, 713-723 (2010).

23. Sauer, G. et al. Proteome analysis of the human mitotic spindle. Mol. Cell Proteomics 4, 35-43 (2005).

24. Daum, J. R. et al. Ska3 is required for spindle checkpoint silencing and the maintenance of chromosome cohesion in mitosis. Curr. Biol. 19, 1467-1472 (2009).

25. Gaitanos, T. N. et al. Stable kinetochore-microtubule interactions depend on the Ska complex and its new component Ska3/C13Orf3. EMBO J. 28, 1442-1452 (2009).

26. Welburn, J. P. et al. The human kinetochore Skal complex facilitates microtubule depolymerization-coupled motility. Dev. Cell 16, 374-385 (2009).

27. Hanisch, A., Sillje, H. H. \& Nigg, E. A. Timely anaphase onset requires a novel spindle and kinetochore complex comprising Skal and Ska2. EMBO J. 25, 5504-5515 (2006).

28. Raaijmakers, J. A., Tanenbaum, M. E., Maia, A. F. \& Medema, R. H. RAMA1 is a novel kinetochore protein involved in kinetochore-microtubule attachment. J. Cell Sci. 122, 2436-2445 (2009).

29. Theis, M. et al. Comparative profiling identifies C13orf3 as a component of the Ska complex required for mammalian cell division. EMBO J. 28, 1453-1465 (2009). 
30. Chan, Y. W., Jeyaprakash, A. A., Nigg, E. A. \& Santamaria, A. Aurora B controls kinetochore-microtubule attachments by inhibiting Ska complexKMN network interaction. J. Cell Biol. 196, 563-571 (2012).

31. Schmidt, J. C. et al. The kinetochore-bound Skal complex tracks depolymerizing microtubules and binds to curved protofilaments. Dev. Cell 23, 968-980 (2012).

32. Ohta, S. et al. The protein composition of mitotic chromosomes determined using multiclassifier combinatorial proteomics. Cell 142, 810-821 (2010).

33. Alushin, G. M. et al. The Ndc80 kinetochore complex forms oligomeric arrays along microtubules. Nature 467, 805-810 (2010).

34. Alushin, G. M. et al. Multimodal microtubule binding by the Ndc80 kinetochore complex. Nat. Struct. Mol. Biol. 19, 1161-1167 (2012).

35. Wang, H. W. et al. Architecture of the Dam1 kinetochore ring complex and implications for microtubule-driven assembly and force-coupling mechanisms. Nat. Struct. Mol. Biol. 14, 721-726 (2007).

36. Jeyaprakash, A. A. et al. Structural and functional organization of the Ska complex, a key component of the kinetochore-microtubule interface. Mol. Cell 46, 274-286 (2012)

37. la Cour, T. et al. Analysis and prediction of leucine-rich nuclear export signals. Protein Eng. Des. Sel. 17, 527-536 (2004).

38. Haering, C. H. et al. Structure and stability of cohesin's Smc1-kleisin interaction. Mol. Cell 15, 951-964 (2004).

39. Gajiwala, K. S. \& Burley, S. K. Winged helix proteins. Curr. Opin. Struct. Biol. 10, 110-116 (2000).

40. Zheng, N. et al. Structure of the Cul1-Rbx1-Skp1-F boxSkp2 SCF ubiquitin ligase complex. Nature 416, 703-709 (2002).

41. Kumar, A. et al. Structure of a novel winged-helix like domain from human NFRKB protein. PLoS One 7, e43761 (2012).

42. Gloyd, M., Ghirlando, R. \& Guarne, A. The role of MukE in assembling a functional MukBEF complex. J. Mol. Biol. 412, 578-590 (2011).

43. Lampson, M. A. \& Cheeseman, I. M. Sensing centromere tension: Aurora B and the regulation of kinetochore function. Trends Cell Biol. 21, 133-140 (2011).

44. Ciferri, C. et al. Architecture of the human ndc80-hecl complex, a critical constituent of the outer kinetochore. J. Biol. Chem. 280, 29088-29095 (2005).

45. Westermann, S., Drubin, D. G. \& Barnes, G. Structures and functions of yeast kinetochore complexes. Annu. Rev. Biochem. 76, 563-591 (2007).

46. Cooper, J. R. \& Wordeman, L. The diffusive interaction of microtubule binding proteins. Curr. Opin. Cell Biol. 21, 68-73 (2009).

47. Maddox, P. S., Oegema, K., Desai, A. \& Cheeseman, I. M. 'Holo'er than thou: chromosome segregation and kinetochore function in C. elegans. Chromosome Res. 12, 641-653 (2004).

48. Maurer, S. P., Fourniol, F. J., Bohner, G., Moores, C. A. \& Surrey, T. EBs recognize a nucleotide-dependent structural cap at growing microtubule ends. Cell 149, 371-382 (2012).

49. Collaborative Computational Project, Number 4. The CCP4 suite: programs for protein crystallography. Acta Crystallogr. D Biol. Crystallogr. 50, 760-763 (1994).

50. Adams, P. D. et al. PHENIX: a comprehensive Python-based system for macromolecular structure solution. Acta Crystallogr. D Biol. Crystallogr. 66, 213-221 (2010).

51. Emsley, P. \& Cowtan, K. Coot: model-building tools for molecular graphics. Acta Crystallogr. D Biol. Crystallogr. 60, 2126-2132 (2004).

52. Case, D. A. et al. AMBER 12 (University of California, San Franciso, 2012).

53. Humphrey, W., Dalke, A. \& Schulten, K. VMD: visual molecular dynamics. J. Mol. Graph. 14, 27-28 (1996).

54. Schneider, C. A., Rasband, W. S. \& Eliceiri, K. W. NIH Image to ImageJ: 25 years of image analysis. Nat. Methods 9, 671-675 (2012).

55. Rappsilber, J., Ishihama, Y. \& Mann, M. Stop and go extraction tips for matrixassisted laser desorption/ionization, nanoelectrospray, and LC/MS sample pretreatment in proteomics. Anal. Chem. 75, 663-670 (2003).
56. Chen, Z. A. et al. Architecture of the RNA polymerase II-TFIIF complex revealed by cross-linking and mass spectrometry. EMBO J. 29, 717-726 (2010).

57. Braun, N. et al. Multiple molecular architectures of the eye lens chaperone alphaB-crystallin elucidated by a triple hybrid approach. Proc. Natl Acad. Sci. USA 108, 20491-20496 (2011).

58. Soares, D. C. et al. The mitosis and neurodevelopment proteins NDE1 and NDEL1 form dimers, tetramers, and polymers with a folded back structure in solution. J. Biol. Chem. 287, 32381-32393 (2012).

59. Cox, J. \& Mann, M. MaxQuant enables high peptide identification rates, individualized p.p.b.-range mass accuracies and proteome-wide protein quantification. Nat. Biotechnol. 26, 1367-1372 (2008).

60. Sillje, H. H., Nagel, S., Korner, R. \& Nigg, E. A. HURP is a Ran-importin betaregulated protein that stabilizes kinetochore microtubules in the vicinity of chromosomes. Curr. Biol. 16, 731-742 (2006).

61. Elbashir, S. M. et al. Duplexes of 21-nucleotide RNAs mediate RNA interference in cultured mammalian cells. Nature 411, 494-498 (2001).

\section{Acknowledgements}

We thank Elena Conti for her generous support during the initial stages of the project We thank the staff of Diamond Light Source for assistance during data collection and staff of Edinburgh protein production facility for the access. We furthermore thank Zhuo Angel Chen, Lauri Peil, Lutz Fischer, Colin Combe, Salman Tahir and Jimi-Carlo Bukowski-Wills for support during the crosslink/MS analysis. A.M. acknowledges the computational facilities of Bioinformatics Center, NII for providing access to their computational resources. We also thank Bill Earnshaw, Kevin Hardwick, Sutapa Chakrabarti, Lakxmi Subramanian and Esben Lorentzen for critical reading of the manuscript. The Wellcome Trust generously supported this work through a Wellcome Trust Career Development Grant to A.A.J. (095822), a Senior Research Fellowship to J.R. (084229), a Wellcome Trust Centre Core Grant (092076) and an instrument grant (091020). A.A.J. also acknowledges the support by the EC for the Marie-Curie career integration grant (FP7). A.S. and E.A.N. acknowledge support from the University of Basel, the Swiss Cancer League (02657-08-2010) and the Swiss National Science Foundation (310030B_149641). A.M. thanks the Department of Biotechnology (DBT), India, for funding.

\section{Author contributions}

A.A.J. conceived and designed the project. M.A.A., B.M., A.A.J., A.S., J.Z., C.P.-H., A.M., U.J. and P.M.R. performed the experiments and analysis. J.R. and E.A.N. provided resources for crosslinking/MS and in vivo experiments, respectively. A.A.J., M.A.A., B.M., A.S., J.Z., A.M., J.R. and E.A.N. wrote the manuscript.

\section{Additional information}

Accession codes: Coordinates and structure factors have been deposited in the protein data bank under accession codes $4 \mathrm{C} 9 \mathrm{Y}$ (C222 $2_{1}$ form) and 4CA0 ( $3_{2}$ form). Supplementary Information accompanies this paper at http://www.nature.com/nature communications

Competing financial interests: The authors declare no competing financial interests.

Reprints and permission information is available online at http://npg.nature.com/ reprintsandpermissions/

How to cite this article: Abad, M. A. et al. Structural basis for microtubule recognition by the human kinetochore Ska complex. Nat. Commun. 5:2964 doi: 10.1038/ncomms3964 (2014).

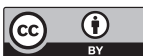

This article is licensed under a Creative Commons Attribution 3.0 Unported Licence. To view a copy of this licence visit http:// creativecommons.org/licenses/by/3.0/
} 